\title{
II. Die Diskussion um die Einbeziehung West- deutschlands in das European Recovery Program 1947 bis März 1948
}

Als im Frühjahr 1947 in den Vereinigten Staaten konkrete Planungen zur Überwindung der europäischen Krise anliefen, stimmten die maßgeblichen amerikanischen Europaund Deutschlandexperten im Prinzip darin überein, daß Deutschland in die Bemühungen um den Wiederaufbau Europas einbezogen werden müsse. Aufgrund der früheren zentralen Stellung des Landes im europäischen Wirtschaftsorganismus sprachen dafür schon allein pragmatisch-ökonomische Erwägungen. Da jedoch eine Restauration der einstigen Machtposition Deutschlands unbedingt verhindert werden sollte, kam es darauf an, den Wiederaufbau so zu dimensionieren, daß wirtschaftliche und politische Notwendigkeiten in Einklang gebracht wurden. Als sich der „Kalte Krieg“ zuspitzte, wuchs die Dringlichkeit einer entsprechenden Lösung, zumal der westliche Teil des besetzten Landes nun auch aus politischen und militärischen Gründen gebraucht wurde. Die neue internationale Konstellation stellte insbesondere die westeuropäischen Nachbarn Deutschlands vor schwierige Probleme. Während beispielsweise die Niederlande und Belgien ihren handelspolitischen Interessen bald Priorität einräumten und den amerikanischen Kurs unterstützten, achtete die französische Regierung energisch darauf, daß in der allgemeinen Wiederaufbaubegeisterung der Vorrang der ehemaligen Kriegsgegner des Deutschen Reiches gewahrt blieb. Noch wichtiger als das Tempo der Rekonstruktionsbemühungen war freilich, vor allem aus deutscher Sicht, deren Ziel: ${ }^{1}$ Wurde Deutschland lediglich als Rohstofflieferant gebraucht, oder sollte es wieder den traditionellen europäischen Spitzenplatz als Fertigwarenexporteur einnehmen? Unter der vagen Parole der Beteiligung am europäischen Wiederaufbau waren nämlich beide Rollen denkbar, wie die gegensätzlichen Überlegungen insbesondere links und rechts des Rheins verdeutlichten. Nun mußte sich zeigen, welche dieser beiden Rollen den außen-, wirtschafts- und sicherheitspolitischen Kalkulationen, Erwartungen, Ambitionen und Möglichkeiten der einzelnen Akteure besser gerecht wurde.

\footnotetext{
1 Dieser wichtige Aspekt wird in der Literatur zum ERP und zur Rolle Deutschlands kaum beachtet. Zu den wenigen Ausnahmen zählt Winfried W. Kretzschmar, Auslandshilfe als Mittel der Außenwirtschafts- und Außenpolitik. Eine Studie über die amerikanische Auslandshilfe von 1945 bis 1956 unter Berücksichtigung sowohl wirtschaftlicher als auch praktisch-politischer Gesichtspunkte, München 1964, bes. S. 189. Aus zeitgenössischer Sicht: Deutschlands Beitrag zum Marshall-Plan. Ausgewählte Kapitel aus den Harriman- und Herter-Reports, mit einer Einl. v. Fritz Baade, Hamburg 1948, bes. S. 6 f.
} 


\section{Amerikanische Pläne und Erwartungen}

Bereits in den ersten einschlägigen Überlegungen zu einem europäischen Hilfsprogramm, die nach der Moskauer Außenministerkonferenz vom Frühjahr 1947 in der amerikanischen Administration entwickelt wurden, tauchte Deutschland als eines der wichtigsten Teilnehmerländer auf. Daß dies, nur zwei Jahre nach Kriegsende, nicht überall selbstverständlich gewesen sein dürfte, war gewiß nur einer der Gründe für das amerikanische Insistieren auf der Einbeziehung des besetzten Landes. In erster Linie kam darin die Überzeugung der maßgeblichen außenpolitischen Experten zum Ausdruck, daß eine Überwindung der wirtschaftlichen Stagnation und der politischen Schwierigkeiten in Europa ohne einen substantiellen deutschen Beitrag, also ohne eine zumindest begrenzte Wiederankurbelung der Wirtschaft, kaum oder nur mit unnötigen und möglicherweise nicht wiedergutzumachenden Zeitverlusten gelingen könne. Über die Art dieses Beitrags gingen die Meinungen freilich zunächst noch auseinander.

In seiner Rede in Cleveland am 8. Mai 1947 nannte der damalige Under Secretary of State und spätere Außenminister Dean Acheson Deutschland eines der größten „workshops" in Europa und spielte damit bereits auf die frühere Rolle der deutschen Wirtschaft an. ${ }^{2}$ George F. Kennan, der Leiter des eigens zur Entwicklung langfristiger Perspektiven für die amerikanische Außenpolitik Anfang Mai ins Leben gerufenen Policy Planning Staff, konzentrierte sich in einer ersten Richtlinie für dieses Gremium hingegen auf die Rolle des Ruhrgebietes als Kohlenreservoir Europas. Das Memorandum des Policy Staff vom 23. Mai erwähnte denn auch nur die Steigerung der Kohlenproduktion als vordringlich; andere deutsche Beiträge wurden nicht in Erwägung gezogen. ${ }^{3}$ Intern entwickelten einzelne Mitarbeiter dieses Planungsstabes allerdings weitergehende Vorschläge. In einer gemeinsam mit Harold Van B. Cleveland und Ben T. Moore verfaßten Denkschrift vom Mai 1947 verknüpfte Charles P. Kindleberger, der für die „deutschen“ Teile verantwortlich zeichnete, kurz- und längerfristige Ziele zu einem Programm, welches die Wiederherstellung der früheren europäischen Außenhandelsstrukturen propagierte. Zwar räumte er der Kohlenförderung zusammen mit dër Verbesserung der Lebensmittelversorgung Priorität ein, doch mit einer entscheidenden Einschränkung: Bei der Versorgung der europäischen Länder mit Ruhrkohle sollte der deutsche Bedarf nicht gänzlich außer Betracht bleiben. Die amerikanischen Verantwortlichen dürften sich mit der Überwindung dieser beiden Engpässe jedoch nicht zufrieden geben, denn: „The restoration of German trade is important to Europe as a source of goods and an outlet for surpluses." Solange noch kein allgemeines Wiederaufbauprogramm für Europa existiere, müsse als Richtschnur gelten, daß jede Deutschlandpolitik, welche die Rekonstruktion der europäischen Handelsbeziehungen der Vorkriegszeit ernsthaft gefährden könnte,

\footnotetext{
2 Vgl. Dean Acheson, Present at the Creation. My Years in the State Department, New York 1969. Dic Rede ist abgedruckt bei Joseph Marion Jones, The Fifteen Weeks, New York u. a. 1955, S. 274-281.

${ }^{3}$ Memorandum Kennan v. 16. 5. 1947; Kennan an Acheson v. 23. 5. 1947, Policy Planning Staff-Memorandum als Anlage, FRUS 1947, III, S. 220-230. Zu Kennans „Coal for Europe“-Plan vgl. John Gimbel, The Origins of the Marshall-Plan, Stanford, Cal., 1976, bes. S. 203-206. Kennans Darstellung, nach der cr sich für cine "prompte und gründliche Wiederbelebung der deutschen Wirtschaft" eingesetzt hatte, ist also in dieser Angelegenheit und für seine ersten „amtlichen“ Überlegungen nicht zutreffend. Vgl. Gcorge F. Kennan, Memoiren eines Diplomaten, (TB-Ausgabe) München ${ }^{3} 1982$, bes. S. 336-338.
} 
eine schlechte Politik sei. ${ }^{4}$ Das Memorandum William Claytons, ebenfalls eine wichtige Diskussionsgrundlage bei der Vorbereitung des Marshallplans, blieb hinter diesen Überlegungen Kindlebergers zurück: Clayton, im Außenministerium für Wirtschaftsfragen zuständig, plädierte für eine gemeinsame europäische Initiative unter Führung Großbritanniens, Frankreichs und Italiens; der deutsche Beitrag erschöpfte sich für ihn in einer Erhöhung der Kohlenförderung an der Ruhr. ${ }^{5}$ Als sich hohe Beamte des State Department schließlich am 28. Mai 1947 mit Außenminister Marshall trafen, um eine erste Bilanz zu ziehen, war von Deutschland wiederum nur im Zusammenhang mit dem akuten Kohlenmangel in Europa die Rede. 6 Die amerikanischen Planungen konzentrierten sich zunächst offensichtlich auf die Lösung der vordringlichsten Aufgaben; eine Festschreibung der künftigen deutschen Rolle beim wirtschaftlichen Wiederaufbau Europas war damit jedenfalls nicht verbunden.

Außenminister Marshall, der in seiner berühmten Harvard-Rede vom 5. Juni 1947 verständlicherweise auf konkrete Vorschläge an die Adresse einzelner Regierungen verzichtet hatte, war sich der Ressourcen, Möglichkeiten und Probleme der potentiellen Teilnehmer an dem von ihm vorgeschlagenen, gemeinschaftlichen Projekt sehr wohl bewußt. Die Dimensionen des "deutschen Problems“, das auch die innerwestlichen Beziehungen belastete, waren ihm zuletzt während der Moskauer Außenministerkonferenz vor Augen geführt worden.? In einem Telegramm an den amerikanischen Militärgouverneur General Lucius D. Clay vom 19. Juni, in dem er eine Stellungnahme zur Frage eines deutschen Beitrags zum Wiederaufbauprogramm erbat, zeigte sich Marshall deshalb von der Notwendigkeit der Einbeziehung zumindest Westdeutschlands überzeugt: „Effective economic restoration of Western Europe requires assistance which Western Germany can give which in turn requires substantial degree of rehabilitation in Germany." Spezielle Erwartungen, beispielsweise hinsichtlich der Kohlenförderung, meldete er nicht an; auf keinen Fall sollte der „europäische“ Beitrag auf Kosten der raschen Wiederaufrichtung einer "selbsttragenden“ deutschen Wirtschaft gehen. ${ }^{8}$

Mit diesem Vorbehalt versuchte Marshall einem möglichen Einwand Clays von vornherein zu entgehen. Die Bemühungen um die Formulierung eines europaweiten Wiederaufbauprogramms fielen nämlich in eine Phase, in welcher die amerikanische Besatzungspolitik neu definiert wurde. An die Stelle des ursprünglichen Ziels, die deutsche Wirtschaft ohne Rücksicht auf europäische Erfordernisse in ihrer Entfaltung zu hemmen, so der bissige Kommentar Kindlebergers in dem oben erwähnten Memorandum, sei die Absicht getreten, die Bizone innerhalb von drei Jahren von fremder Hilfe unabhängig zu machen, wiederum ohne Rücksicht auf die anderen europäischen Länder. ${ }^{9}$

\footnotetext{
4 Das Memorandum ist auszugsweise wiedergegeben in Charles P. Kindleberger, Marshall Plan Days, Boston u. a. 1987, S. 4-24; die Zitate S. 14, 18 u. 23.

${ }^{5}$ Memorandum Clayton v. 27. 5. 1947, FRUS 1947, III, S. 230-232.

6 Vgl. Summary of Discussion v. 29. 5. 1947, FRUS 1947, III, S. 234-236; Kennan, Memoiren, S. 345 f.

7 Vgl. Forrest C. Pogue, George C. Marshall: Statesman 1945-1959, New York 1987, S. 168-196. Die Harvard-Rede ist abgedruckt in FRUS 1947, III, S. 237-239.

8 Adjutant General, War Dept., an Clay, Ref. No. WX-80548, Institut für Zeitgeschichte/Archiv (künftig IfZA), MF 260, POLAD/784-2; Datierung nach Marshall an Botschaft London v. 20.6. 1947, FRUS 1947, III, S. 263. Telegramme mit der Bitte um Stellungnahmen zu seiner Initiative und zu den „Hilfsmöglichkeiten“ der einzelnen Länder sandte Marshall an verschiedene Botschaften in Westeuropa. In dem Telegramm beispielsweise an die Pariser Botschaft wird allerdings die Einbeziehung Deutschlands nicht erwähnt; vgl. Marshall an Botschaft Paris v. 12. 6. 1947, FRUS 1947, III, S. 249-251.

9 Vgl. Kindleberger, Marshall Plan Days, S. 4.
} 
Dieses Urteil war allerdings nicht ganz fair und spiegelte gewiß auch die Spannungen zwischen State Department und War Department wider. Immerhin hatte der von Kriegsminister Robert P. Patterson angeregte Hoover-Report über die deutschen Wirtschaftsprobleme europäische Belange nicht unberücksichtigt gelassen. Freilich war sein Ansatz auf Deutschland konzentriert; mit der Erholung der deutschen Wirtschaft, so die Erwartung des früheren US-Präsidenten, würden sich viele Schwierigkeiten in Europa leichter überwinden lassen. Er plädierte deshalb dafür, verstärkt die Schwerindustrie aufzubauen, damit Deutschland an seine "ein Jahrhundert hindurch" wahrgenommene Aufgabe als „eines der großen europäischen Zentren für die Produktion von Kapitalgütern" - er erwähnte im einzelnen Baumaterialien, Fabrikanlagen, Eisenbahnbedarf, elektrische und schwere Maschinen - anknüpfen könne. ${ }^{10}$ Selbst wenn manche der Empfehlungen Hoovers zunächst nur geringe Realisierungschancen besaßen, stellte sein Bericht zweifelsohne eines der ersten aufsehenerregenden Plädoyers für einen Kurswechsel in der amerikanischen Deutschlandpolitik dar.

Einer der entschiedenen Verfechter des neuen Kurses unter den Verantwortlichen vor Ort und in Washington war General Clay. Noch Anfang Mai 1947 hatte er Außenminister Marshall erläutert, wie er sich die Wiederankurbelung der bizonalen Wirtschaft vorstellte. Die Bizone durfte seiner Meinung nach nicht zum Kohlenexporteur degradiert werden, vielmehr mußte sie in die Lage versetzt werden, für die Ausfuhr produzieren und gewinnbringende Handelsbeziehungen aufbauen zu können. Für gegensätzliche Forderungen und Interessen der Nachbarländer brachte er zwar Verständnis auf, doch war er nicht bereit, deswegen weitere wirtschaftliche Opfer zu bringen, die letztlich doch nur zu Lasten der amerikanischen und britischen Staatskasse gehen würden. ${ }^{11} \mathrm{Er}-$ forderte der Marshallplan eine Abkehr von dieser eben erst in die Wege geleiteten, bizonen-orientierenden Politik, oder war es möglich, den Wiederaufbau in Deutschland mit den übergreifenden europäischen Plänen in Einklang zu bringen?

Bereits am 24. Juni lag eine erste interne Stellungnahme aus dem Büro des Politischen Beraters Clays, Robert D. Murphy, vor. Ihr Autor, Wesley C. Haraldson, hielt einen deutschen Beitrag zum europäischen Wiederaufbau nicht nur für wünschenswert, sondern auch für möglich. Seine Vorschläge konzentrierten sich auf ein Bündel von Maßnahmen zur Ankurbelung der Binnenwirtschaft sowie auf die Wiederbelebung des AuBenhandels. Zu diesem Bündel zählten die Festsetzung neuer, „endgültiger“ Obergrenzen für die Industrieproduktion, die Steigerung der Kohlenförderung, eine Währungsreform, die Erhöhung der landwirtschaftlichen Erzeugung, die Instandsetzung des Transportwesens, sowie, als politische „Beigabe“, die Revision des Entnazifizierungsprogramms. Neben der Überwindung akuter Engpässe hatten diese Vorschläge also vor allem das Ziel, stabile und kalkulierbare Bedingungen für die notwendigen wirtschaftlichen Aktivitäten zu schaffen. Nicht minder bedeutsam erschien Haroldson die Normalisierung der Handelsbeziehungen. Zu diesem Zweck regte er in erster Linie die Bereit-

${ }^{10}$ Hoover-Bericht Nr. 3 v. 18. 3. 1947, in deutscher Übersetzung abgedruckt in Gustav Stolper, Die deutsche Wirklichkeit. Ein Beitrag zum künftigen Frieden Europas, Hamburg 1949, S. 336-347, das Zitat S. 344. Vgl. auch Gimbel, Origins, S. 179-186.

11 Vgl. Clay an Marshall v. 2. 5. 1947, zit. n. The Papers of General Lucius D. Clay. Germany 1945-1949 (künftig Clay Papers), Vol. I, ed. by Jean Edward Smith, Bloomington-London 1974, S. 346-349. Vgl. auch Wolfgang Krieger, General Lucius D. Clay und die amerikanische Deutschlandpolitik 1945-1949, Stuttgart 1987, S. $278 \mathrm{f}$. 
stellung von Export-Import-Krediten durch Privatbanken, Regierungen oder Regierungsagenturen an, mit deren Hilfe der wegen des Dollarmangels auf „essentials“ beschränkte Handel ausgeweitet werden sollte: „With increased appropriated funds or proper credit arrangements Germany can, once again, trade principally with Europe and the Scandinavian countries where it can render most effective aid." Weiter empfahl er, ausländische Investitionen wieder zuzulassen, die deutschen Zolltarife zu senken und in den bestehenden europäischen Organisationen wie der Economic Commission for Europe (ECE) und der European Coal Organization (ECO) ernsthaft mitzuarbeiten. Da Deutschland nicht in der Lage sei, zur Wiedergenesung Europas Finanzhilfen oder Nahrungsmittellieferungen beizusteuern, so das Fazit, bleibe nur die Möglichkeit, die europäischen Länder mit den Erzeugnissen seiner Industrie und Bergwerke sowie mit Dienstleistungen im Transportsektor zu versorgen.12

In einem zweiten Memorandum befaßte sich Louis A. Wiesner, im Stab des Politischen Beraters für Arbeiterfragen zuständig, mit den politischen Aspekten einer Teilnahme Deutschlands am Marshallplan. Während Außenminister Marshall in seinem oben zitierten Telegramm an Clay offensichtlich unterstellte, daß die UdSSR das Kooperationsangebot ablehnen werde - zu einem Zeitpunkt, als die Pariser Verhandlungen zwischen Bidault, Bevin und Molotow noch nicht einmal begonnen hatten -, bezog Wiesner die Möglichkeit einer positiven sowjetischen Antwort in seine Überlegungen ein. Die von ihm vorgeschlagenen politisch-ökonomischen Minimalbedingungen für eine Zusammenarbeit ließen jedoch erkennen, daß er die UdSSR zu einem „Nein“ provozieren wollte: Schon die Forderung nach echter wirtschaftlicher Integration des ganzen Kontinents unter der Kontrolle einer effektiven, supranationalen Organisation mußte für die Sowjetunion problematisch sein, stand aber wenigstens noch in erkennbarem Zusammenhang mit dem geplanten Hilfsprogramm; anders verhielt es sich mit Punkten wie „Befreiung der drei baltischen Staaten“ oder „Ostverschiebung der provisorischen deutsch-polnischen Grenze“, die eindeutig politisch motiviert waren. Sollte die Sowjetunion diese Bedingungen nicht akzeptieren - und daran konnte es angesichts des sich verschärfenden „Kalten Krieges“ keinen Zweifel geben -, dann sollte der Westen damit beginnen, die Kommunisten in Westdeutschland und Westeuropa ebenso hart anzufassen, wie dies Nicht-Kommunisten in Ostdeutschland und Osteuropa durch sowjetische Stellen widerfuhr. Besondere Sorge bereitete Wiesner der vermeintlich große kommunistische Einfluß im Ruhrgebiet; Schätzungen zufolge waren etwa 80000 Bergleute KPD-Mitglieder. Um die Kohlenförderung kontinuierlich steigern zu können, müsse dieses Potential zumindest neutralisiert werden: „A bit of terror against KPD leaders might help in this regard." Der Gefahr einer, wie er glaubte, zeitweiligen Spaltung Europas war er sich wohl bewußt, doch müßten die USA dessenungeachtet alle Kraft auf den Wiederaufbau und die Demokratisierung Westeuropas einschließlich Westdeutschlands konzentrieren. ${ }^{13}$

In Clays Antwort an Marshall, Patterson und Marineminister Forrestal tauchten die

12 Memorandum Haraldson: German contribution to European Economic Recovery v. 24. 6. 1947, IfZA, MF 260, POLAD/784-2. - Zur ECE vgl. David Wightman, Economic co-operation in Europe. A study of the United Nations Economic Commisson for Europe, New York/Toronto 1956; zur ECO Albrecht Weber, Geschichte der internationalen Wirtschaftsorganisation, Wiesbaden 1983, S. 102.

13 Wiesner an Murphy v. 25. 6. 1947, IfZA, MF 260, POLAD/784-2. 
- selbst nach eigenem Eingeständnis - teilweise allzu radikalen Empfehlungen Wiesners nicht auf. Der amerikanische Militärgouverneur konzentrierte sich auf die wirtschaftlichen Probleme, ohne allerdings die politischen Hindernisse für einen angemessenen deutschen Beitrag zum Wiederaufbau, beispielsweise die fehlende Wirtschaftseinheit oder die alliierten Restriktionen, gänzlich außer acht zu lassen. Nachkriegsdeutschland hatte bis dahin, abgesehen von den Kohlenexporten, beim Wiederaufbau der europäischen Wirtschaft keine bedeutende Rolle gespielt. Um diesen Zustand zu ändern, kam es zunächst darauf an, die Lebensmittelversorgung zu verbessern. Außer Kohle, die jedoch für den eigenen wirtschaftlichen Wiederaufbau dringend gebraucht wurde, und Holz konnte eine leistungsfähige Bizone nämlich auch Textilien, Farbstoffe, pharmazeutische und chemische Stoffe, Ersatzteile für alle Arten von Maschinen sowie landwirtschaftliche und andere Maschinen liefern. Clay bekräftigte seinen Standpunkt, daß Deutschland zunächst nur die allernotwendigsten Güter in Europa kaufen könne und daß in allen Fällen, in denen keine "essentials" im Austausch für deutsche Exporte zur Verfügung stünden, weiterhin in Dollar bezahlt werden müsse. Sehr viel hing seiner Meinung nach von der Kooperationsbereitschaft der übrigen Länder ab: „The other European nations will need to assist recovery by recognizing that the war is over and by reestablishing the type of trade, communications and travel with Germany that are appropriate to peacetime." Besonders heikel war in diesem Zusammenhang die Frage der Prioritäten. Zwar stellte Clay den allgemeinen Konsens - Vorrang für die ehemals von Deutschland besetzten Staaten - nicht offen in Frage, doch machte er unmißverständlich deutlich, daß der Vorsprung nicht zu groß werden durfte: "Germany must be given hope to early equality in treatment and not relegated indefinitely to an inferior status." Robert Murphy, der zwei Tage später ein eigenes, noch mehr ins Detail gehende Telegramm an Marshall richtete, schloß sich dieser Argumentation Clays weitgehend an. ${ }^{14}$

Früher und entschiedener als nach dem Ersten Weltkrieg drängte die westliche Führungsmacht also auf einen tendenziell gleichberechtigten Einschluß Deutschlands in das Rekonstruktions- und Stabilisierungsprogramm für Europa. Aus dem Mißerfolg in den zwanziger Jahren wurden offensichtlich Lehren gezogen. Dieses "neue Denken“ ist freilich nicht allein mit dem Hinweis auf den Lernprozeß der amerikanischen Deutschlandund Europapolitiker zu erklären. Ebenso wichtig war die veränderte internationale Lage, der sich rasch verschärfende Konflikt mit der Sowjetunion. ${ }^{15} \mathrm{Um}$ in dieser Auseinandersetzung bestehen zu können, schien zumindest das westdeutsche Potential unverzichtbar. Das Bemühen, den Wiederaufbau in Deutschland oder wenigstens in der Bizone mit dem im Entstehen begriffenen europäischen Programm abzustimmen, war den Stellungnahmen Clays und seiner Mitarbeiter anfangs deutlich anzumerken; ebenso offensichtlich war aber auch, daß sie nur wenig Neigung zeigten, der Rekonstruktion der anderen Volkswirtschaften auf Dauer Priorität einzuräumen. Außerdem wehrten sie sich dagegen, daß der deutsche Beitrag in erster Linie darin bestehen sollte, Europa mit Ruhrkohle zu versorgen; ihre Vorstellungen orientierten sich, mehr oder weniger ausge-

${ }^{14}$ Clay an Secretaries of State, War and Navy v. 28. 6. 1947, Clay Papers I, S. 377-381; Murphy an Secretary of State v. 30. 6. 1947, FRUS 1947, II, S. 977-982; vgl. auch Gunther Mai, Der Alliierte Kontrollrat in Deutschland 1945-1948. Alliierte Einheit - deutsche Teilung?, München 1995, S. 437.

15 Diesen Aspekt betont Melvyn P. Leffler, A Preponderance of Power. National Security, the Truman Administration, and the Cold War, Stanford, Cal., 1992, S. 151-164, ganz besonders. 
prägt, an der früheren Rolle des Deutschen Reichs im europäischen Wirtschaftsgeflecht. In dieser Grundsatzfrage stimmten die Experten der Militärregierung und des State Department sicherlich überein, wenngleich letztere in dem Bestreben, zunächst die akuten Versorgungsengpässe in Europa zu meistern, ihr Hauptaugenmerk auf die Steigerung der Förderung und des Exports der Ruhrkohle richteten. Selbst in der Frage der Bevorzugung der ehemaligen Kriegsgegner Deutschlands zeichnete sich eine Annäherung der Standpunkte ab, weil auch unter den Planern des Außenministeriums die Überzeugung wuchs, daß wegen des großen Rückstands der wirtschaftlichen Entwicklung in den Besatzungszonen in absehbarer Zeit ein Ein- oder gar Überholen der Nachbarländer ohnehin nicht zu befürchten sei. ${ }^{16}$ Freilich war im State Department nach wie vor die Bereitschaft vorhanden, in dieser brisanten Angelegenheit auf die Regierungen und die Öffentlichkeit in Europa, insbesondere in Frankreich, Rücksicht zu nehmen, während Clays Geduld langsam zu Ende ging.

\section{Erste Reaktionen in Westeuropa}

Die amerikanische Absicht, Deutschland in das geplante Wiederaufbauprogramm einzuschließen, stieß in den westeuropäischen Regierungszentralen auf ein geteiltes Echo. Zwar zweifelte kaum einer unter den maßgebenden Politikern und Wirtschaftsexperten in London, Paris oder Den Haag an, daß die deutsche Wirtschaft ein integraler Bestandteil der europäischen Wirtschaft war und eine Vernachlässigung dieser Kernregion somit die wirtschaftliche Wiederbelebung Europas zumindest verzögern, wenn nicht gar ganz verhindern würde; strittig war jedoch, ob der ehemalige Kriegsgegner von Anfang an "gleichberechtigt" in ein solches Sanierungsprogramm einbezogen werden sollte.

Die britische Regierung, die für Großbritannien ohnehin eine Sonderrolle in dem geplanten Hilfsprogramm beanspruchte, hatte keine grundsätzlichen Einwände gegen eine Gleichbehandlung Deutschlands mit den übrigen potentiellen Teilnehmerländern. Für eine begrenzte und kontrollierte Wiederbelebung der deutschen Wirtschaft, und zwar nicht allein des Ruhrbergbaus, waren maßgebliche Politiker und Planer schon gegen Ende des Krieges eingetreten ${ }^{17}$; die Kosten, die Großbritannien als Besatzungsmacht erwuchsen, taten ein übriges. Auf der Moskauer Außenministerkonferenz vom Frühjahr 1947 unterstützte Bevin seinen amerikanischen Kollegen deshalb in seinen Bemühungen, eine neue, weniger restriktive alliierte Wirtschaftspolitik durchzusetzen: die Produktionsverbote und -beschränkungen sollten zumindest soweit gelockert werden, daß die Deutschen die nötigen Einfuhren ohne fremde Hilfe, nämlich durch eigene Exporte,

${ }^{16}$ In einem Report des Policy Planning Staff vom 23. Juli 1947 mit dem Titel „Certain Aspects of the European Recovery Problem from the United States Standpoint" heißt es dazu: "The present situation in the U.S.-U.K. zones, however, in which industrial production is less than half of pre-war, food supplies are considerably below the minimum requirements for health and efficiency, and foreign trade is only a trickle of its former dimensions, represents a degree of retardation in the recuperation of German production far greater than any reasonable system of priorities would warrant. Between this point and a point where it could be claimed that the interests of German economy were being favored over those of Germany's present neighbors, there lies a wide gap most of which must be filled before general European recovery can become a reality." The State Department Policy Planning Staff Papers (künftig: PPSP) 1947, Vol. I, New York-London 1983, S. 26-68, hier S. 56.

17 Vgl. Albrecht Tyrell, Großbritannien und die Deutschlandplanung der Alliierten 1941-1945, Frankfurt a. M. 1987 , bes. S. $484-605$. 
bezahlen konnten. ${ }^{18}$ Das Interesse an einer Reduzierung der Besatzungskosten bestimmte also die britische Haltung in erster Linie, weniger die Sorge um das wirtschaftliche und politische Schicksal Europas und schon gar nicht die Absicht, einen potentiellen Konkurrenten auf dem Weltmarkt von vornherein auszuschalten. Letztere Aussicht mochte für manche Beamte des Foreign Office oder des Board of Trade, mehr noch für die britische Industrie, durchaus verlockend gewesen sein - für die maßgeblichen Deutschlandpolitiker spielte dieses Motiv indessen keine Rolle. ${ }^{19}$

Für die kleineren nord- und westeuropäischen Länder war es vor allem der Ausfall eines der wichtigsten früheren Märkte - der sich inzwischen bereits nachteilig bemerkbar machte -, der eine Beteiligung Deutschlands wünschenswert erscheinen ließ. Etwa 21 Prozent der Einfuhren und 17 Prozent der Ausfuhren hatte beispielsweise Schweden in den letzten Jahren vor Beginn des Zweiten Weltkrieges mit dem Deutschen Reich abgewickelt: importiert wurden insbesondere Erzeugnisse des Maschinenbaus, der Stahl-, Textil- und chemischen Industrie. Solche wirtschafts- und handelspolitischen Gründe ließen auch in anderen skandinavischen Ländern den Wunsch nach einem Wiedererstarken des früheren Handelspartners reifen. ${ }^{20}$ Ähnlich war die Situation in den Beneluxstaaten, die mit dem östlichen Nachbarn ebenfalls intensive Handelsbeziehungen unterhalten hatten. Die niederländische Regierung beispielsweise hatte sich deshalb schon im Dezember 1946 mit einem Memorandum über Möglichkeiten zur wirtschaftlichen Wiederaufrichtung Deutschlands an die Regierungen in Washington und London gewandt. Sie erinnerte darin an die in der Vergangenheit stets sehr engen niederländisch-deutschen Wirtschaftsbeziehungen: Im Gegenzug für beträchtliche Importe von Maschinen, Ersatzteilen und Ausrüstungsgütern waren Veredelungsaufträge an die deutsche Industrie vergeben worden; niederländische Handelsgesellschaften und Banken hatten einen großen Teil der deutschen Rohstoffimporte abgewickelt, und die Häfen von Rotterdam und Amsterdam hatten überdies als Umschlagplatz für den deutschen Überseehandel gedient. Um die den Wiederaufbau im eigenen Land gefährdende wirtschaftliche Stagnation in Deutschland zu überwinden, hatte die Regierung in Den Haag bereits eine Gesellschaft gegründet, welche den Kauf von Rohstoffen, die Veredelung im Nachbarland und den Reexport finanzieren sollte; in Ermangelung ausreichender Mittel wurden nun zusätzliche alliierte Kredite für dieses Vorhaben erbeten. ${ }^{21}$ Wegen dieser ohnehin vorhandenen, nicht aus Sympathie für die Deutschen, sondern aus wirtschaftlichen Zwängen resultierenden Bereitschaft zur konkreten Hilfe wäre alles andere als eine positive Reaktion auf die amerikanische Initiative eine Überraschung gewesen. Die Niederlande

${ }_{18}$ Vgl. Alan Bullock, Ernest Bevin. Foreign Secretary 1945-1951, Oxford-New York 1985 (PaperbackAusgabe), S. 378.

19 Vgl. Ian Turner, British Policy Towards German Industry, 1945-9: Reconstruction, Restriction or Exploitation?, in: ders. (Hrsg.), Reconstruction in Postwar Germany. British Occupation Policy and the Western Zones, 1945-1955, Oxford u. a. 1989, S. 67-91.

${ }^{20} \mathrm{Zu}$ Schweden vgl. Martin Fritz, Schweden und der westdeutsche Markt 1945-1955, in: Herbst/Bührer/ Sowade, Marshallplan, S. 99-117; zur Haltung der sozialdemokratischen Parteien Skandinaviens Klaus Misgeld, Sozialdemokratie und Außenpolitik in Schweden. Sozialistische Internationale, Europapolitik und die Deutschlandfrage 1945-1955, Frankfurt a. M./New York 1984, bes. S. 88 u. 99.

${ }^{21}$ Memorandum concerning a possible Netherlands contribution to the economic restoration of Germany, mit Begleitschreiben an Acting Secretary of State (Eingangsstempel Dept. of State v. 12. 12. 1946), National Archives, Washington, Record Group (künftig NA, RG), 59, 862.50/12-746. 
gehörten denn auch, neben Belgien und Luxemburg, zu den eifrigsten Fürsprechern einer gleichberechtigten Beteiligung des östlichen Nachbarn. ${ }^{22}$

Widerstand regte sich vor allem aus Frankreich. „Nous d'abord, l'Allemagne après“ auf diese ebenso schlichte wie einprägsame und damit publikumswirksame Formel brachte eine französische Zeitung diese nicht nur auf das gaullistische und kommunistische Lager beschränkte Stimmung. ${ }^{23} \mathrm{Zwar}$ war auch Frankreich auf den Handel mit dem Nachbarn jenseits des Rheins angewiesen, Priorität genossen aber sicherheits- und machtpolitische Interessen. Im Kern ging es darum, die deutsche Vormachtstellung in Europa ein für allemal zu brechen. Diesem Ziel diente auch der sogenannte MonnetPlan, der von amerikanischer Seite sogar als mögliches Vorbild für das europaweite Sanierungsprogramm ins Gespräch gebracht wurde. ${ }^{24} \mathrm{Um}$ seine ehrgeizigen Planziffern, insbesondere eine Jahresproduktion von 15 Mio. Tonnen Rohstahl bis 1952, das Saargebiet eingeschlossen, zu erreichen, bedurfte es gewiß eines deutschen „Beitrags“ - allerdings hauptsächlich in Form von Kohle. Wenn nun, wie Regierung und Öffentlichkeit in Frankreich befürchteten, Deutschland in den europäischen Wiederaufbau einbezogen werden würde, waren die eigenen Ziele und Pläne zumindest gefährdet. Aus diesem Grund war Außenminister Georges Bidault fest entschlossen, den Vorrang Frankreichs und der übrigen „alliierten“ Länder zu verteidigen. ${ }^{25}$ In seiner ersten offiziellen Reaktion auf die Marshall-Rede, einer Direktive an die Botschafter in Washington und London vom 10. Juni 1947, konzedierte er zwar beiläufig die Beteiligung Deutschlands, stellte jedoch zugleich klar, daß als Delegierte nur Vertreter der Besatzungsmächte in Frage kämen. Bezeichnenderweise fiel dieser einzige Hinweis im Zusammenhang einer knappen Erörterung des Kohlenproblems. ${ }^{26}$ Allerdings waren zu diesem Zeitpunkt die amerikanischen Absichten noch nicht eindeutig erkennbar, für massive Proteste gab es demzufolge keinen Grund. Dies änderte sich rasch, als vor und während der Pariser Marshallplankonferenz deutlich wurde, daß Deutschland in den Plänen der US-Regierung eine entscheidende Rolle spielte. Daß, nachdem die Sowjetunion das Angebot zur Teilnahme am Marshallplan ausgeschlagen hatte, nur der westliche Teil des besetzten Landes in den Genuß amerikanischer Hilfe kommen würde, dürfte die französischen Besorgnisse kaum gedämpft haben.27

22 Vgl. Albert Kersten, Die Niederlande und die Westintegration der Bundesrepublik. Wirtschaft, Sicherheit und politische Kontrolle, in: Herbst/Bührer/Sowade, Marshallplan, S. 119-138; Milward, Reconstruction, S. $66 \mathrm{f}$.

${ }^{23}$ Vgl. Wilfried Loth, Sozialismus und Internationalismus. Die französischen Sozialisten und die Nachkriegsordnung Europas 1940-1950, Stuttgart 1977, S. 167-175. Das Zitat stammt aus dem „Franc-Tireur" v. 20./21.7. 1947, zit. n. ebenda, S. 171.

${ }^{24}$ Vgl. zum Monnet-Plan Jean Monnet, Erinnerungen eines Europäers, München-Wien 1978, S. 297-336; Philippe Mioche, Le Plan Monnet, Genèse et élaboration 1941-1947, Paris 1987.

25 Vgl. Georges Bidault, Noch einmal Rebell. Von einer Résistance in die andere, Berlin 1966, S. 197.

${ }^{26}$ Vgl. Bidault an Botschaften in Washington und London v. 10.6. 1947, Archives Diplomatiques, Ministère des Affaires Étrangères, Paris (künftig MAE), Y 1944-49, Vol. 228. Der französische Botschafter in Washington, Henri Bonnet, trug Außenminister Marshall am 13.6. 1947 die wesentlichen Punkte dieser Direktive vor; vgl. Mcmorandum of Conservation v. 13. 6. 1947, FRUS 1947, III, S. 251-253.

${ }^{27}$ Zur Haltung der UdSSR gegenüber der amerikanischen Initiative vgl. Wilfried Loth, Die Teilung der Welt. Geschichte des Kalten Krieges 1941-1955, München 1980, S. 172-177; Othmar Nikola Haberl, Die sowjetische Außenpolitik im Umbruchsjahr 1947, in: ders./Lutz Niethammer (Hrsg.), Der Marshall-Plan und die europäische Linke, Frankfurt a. M. 1986, S. 75-96; Mikhail Narinski, Le Plan Marshall et l' URSS, in: Le Plan Marshall et le relèvement économique de l' Europe, hrsg. v. Comité pour l' Histoirc économique et financière de la France, Paris 1993, S. 119-123. Zu den Verhandlungen zwischen Bevin, Bidault und Molotow vgl. FRUS 1947, III, S. 296-308. 
Das deutsche Industriepotential, das ja ohnehin stärker in den Westzonen konzentriert war, empfanden Regierungen und Öffentlichkeit in Europa nämlich nach wie vor als Bedrohung. Auch jene Politiker, Wirtschaftsexperten oder Geschäftsleute, die aus „egoistischen“ wirtschaftlichen Motiven für eine Wiederbelebung der westdeutschen Produktionsmaschinerie eintraten, wollten keine unbegrenzte und unkontrollierte Rekonstruktion und insbesondere keine Restauration der alten deutschen Machtposition. Der Konflikt zwischen Sicherheits- und Wirtschaftsinteressen begann sich erst aufzulösen, als die westeuropäischen Regierungen angesichts der Verschärfung des „Kalten Krieges" neue Feindbilder entwickelten. Dieser Prozeß setzte in den einzelnen Ländern, mit unterschiedlicher Intensität, 1947 ein. Dementsprechend weit war zunächst noch das Spektrum möglicher und erwünschter deutscher Beiträge zum europäischen Wiederaufbau: Es reichte vom „pastoralisierten“ Kohlenexportland bis zur traditionellen Rolle als Anbieter hochwertiger Erzeugnisse der Investitionsgüterindustrie. Verständlicherweise war letztere Perspektive aus deutscher Sicht weitaus attraktiver.

\section{Deutsche Hoffnungen, Wünsche und Ansprüche}

In dem bereits erwähnten Memorandum vom Juni 1947 hatte Kindleberger die Aussichten, die Deutschen zur tatkräftigen Mitarbeit zu motivieren, eher skeptisch beurteilt; ein Appell an das deutsche Nationalgefühl kam schon deshalb nicht in Frage, weil damit in den Nachbarländern nur neue Ängste geweckt worden wären. „There is then little that can be done for the moment to stimulate the Germans to take an active role in the development of a European political and economic recovery." Möglicherweise, so seine Hoffnung, werde sich dies ändern, sobald die Grundlagen für eine eigenverantwortliche politische Führung in Deutschland gelegt waren. ${ }^{28}$ Solche Bedenken mögen aufgrund der heute vorherrschenden, verklärenden Sicht des Marshallplans gänzlich unverständlich erscheinen - völlig unbegründet waren sie damals keineswegs. Zwar überwogen in den ersten deutschen Reaktionen Erleichterung und Freude über den neuen Kurs der amerikanischen Deutschlandpolitik, verbunden mit der Beteuerung, nach Kräften am Wiederaufbau Europas mitarbeiten zu wollen; damit einher ging jedoch, mehr oder weniger deutlich ausgesprochen, die Forderung nach angemessenen Mitspracherechten. Der Marshallplan wurde durchaus als Chance begriffen, nicht nur das Wirtschaftsleben zu reaktivieren, sondern möglicherweise auch die außenpolitische Isolierung zu durchbrechen, doch versuchten maßgebliche politische und wirtschaftliche Kreise in den Westzonen, im sicheren Gefühl der Bedeutung des deutschen Wirtschaftspotentials, ihre Mitarbeit an Bedingungen zu knüpfen. Ähnlich wie ihre Vorgänger in der ersten Hälfte der zwanziger Jahre lehnten sie eine Kooperation um jeden Preis und ohne Gegenleistung $a b$ - während zumindest auf amerikanischer Seite dieses Mal keine Neigung zu erkennen war, die Deutschen selbst über die Teilnahme an den geplanten gemeinsamen Wiederaufbauanstrengungen entscheiden zu lassen.

Das Gremium, das zwei Jahre nach Kriegsende noch am ehesten berufen war, für die große Mehrheit der Deutschen zu sprechen, war der auf Initiative der amerikanischen und britischen Militärregierungen ins Leben gerufene bizonale Wirtschaftsrat. Schon auf

${ }^{28}$ Kindleberger, Marshall Plan Days, S. 9. 
seiner ersten Vollversammlung am 25. Juni 1947 befaßten sich drei der fünf Sprecher der einzelnen Fraktionen in ihren Eröffnungsreden mit den Implikationen des Marshallplans. Friedrich Holzapfel von der CDU hob die Verflechtung der deutschen mit der europäischen Wirtschaft hervor und legte im Namen seiner Partei ein „Bekenntnis zu einer engen Zusammenarbeit" $a b$, wehrte sich aber zugleich massiv gegen den erzwungenen Export von Kohle und Holz, jenen beiden Grundstoffen, die dringend gebraucht wurden, um die eigene Wirtschaft in Gang zu setzen und die Fertigwaren zu produzieren, mit denen die Rohstoff- und Lebensmittelimporte bezahlt werden konnten. Für das Zentrum machte Carl Spiecker darauf aufmerksam, daß die deutsche Wirtschaft "weit mehr exportieren" müsse als früher. Anders als seine Kollegen ging Erwin Schoettle von der SPD explizit auf die Initiative Marshalls ein, ja er würdigte sie als „eines der wichtigsten Ereignisse" der damaligen Zeit. Deutlicher als Holzapfel und Spiecker pochte er aber darauf, die Deutschen müßten „in diese europäische Zusammenarbeit als Subjekt hineingehen, als Teil, der, wenn auch nicht voll gleichberechtigt, aber doch mit der Chance der Gleichberechtigung ausgestattet" sei, weil sie nur so ihren Beitrag zur Lösung der europäischen und ihrer eigenen Probleme leisten könnten. Jedenfalls müsse künftig alles Planen und Denken auf die Eingliederung in den größeren europäischen Rahmen ausgerichtet sein, damit deutsche Stellen auch ohne besondere Aufforderung ihren „bescheidenen, aber unentbehrlichen Beitrag“ zur Bewältigung der gemeinsamen Aufgabe beisteuern könnten. ${ }^{29}$ Auf seiner zweiten Sitzung am 22. Juli verabschiedete der Wirtschaftsrat sogar eine von allen Fraktionen - mit Ausnahme der KPD - gebilligte Erklärung, in der es unter anderem hieß, die deutsche Wirtschaft verfüge „bei entsprechenden Maßnahmen zur Abwehr des jetzt drohenden Zusammenbruchs über wertvolle Produktivkräfte, die einen wesentlichen Beitrag zur Wiederherstellung der europäischen Gesamtwirtschaft leisten“ könnten. Die geplante „organische Eingliederung Deutschlands in den europäischen Wirtschaftsaufbau" ließ nach Auffassung der bürgerlichen Parteien und der SPD allerdings die „Mitwirkung deutscher Sachverständiger aus allen Zonen bei der Ausarbeitung der Pläne notwendig erscheinen. " ${ }^{30}$ In diesem Sinne hatte zwar schon der hessische Ministerpräsident Christian Stock bei General Clay angefragt, ohne jedoch mehr als die vage Zusicherung zu erhalten, daß „deutschen Stellen Gelegenheit gegeben werde, zu dem Plan Stellung zu nehmen, bevor er verwirklicht werde.“ 31

Wie die Stellungnahmen Holzapfels und Schoettles und vor allem die gemeinsame Entschließung des Wirtschaftsrates zeigten, war die Übereinstimmung zwischen CDU

${ }^{29}$ Wörtlicher Bericht über die 1. Vollversammlung am 25. 6. 1947, in: Wörtliche Berichte und Drucksachen des Wirtschaftsrates des Vereinigten Wirtschaftsgebietes 1947-1949, hrsg. v. Institut für Zeitgeschichte und dem Deutschen Bundestag, Wissenschaftliche Dienste, Bd. 2, Nachdruck München/Wien 1977. - Franz Blücher, in der ersten Bundesregierung Minister für den Marshallplan, ging in seiner für die FDP-Fraktion abgegebenen Erklärung mit keinem Wort auf die Rede Marshalls ein. Zur Reaktion deutscher Politiker im Uberblick Klaus Schwabe, German Policy Responses to the Marshall Plan, in: Maier/Bischof, Marshall Plan, S. 225-281.

30 Wörtlicher Bericht über die 2. Vollversammlung v. 22.-24. 7. 1947, S. 4, in: Wörtliche Berichte; vgl. auch Georg Müller, Die Grundlegung der westdeutschen Wirtschaftsordnung im Frankfurter Wirtschaftsrat 1947-1949, Frankfurt a. M. 1982, bes. S. 92. - Ob die gleichfalls zum Ausdruck gebrachte Hoffnung auf ein „einheitliches Zusammenwirken aller europäischen Völker" mehr war als der Versuch, das schlechte deutschlandpolitische Gewissen zu beruhigen, ist schwer zu beurteilen; immerhin verhandelten seit Mitte Juli die teilnahmewilligen Länder in Paris - ohne die Staaten Osteuropas!

${ }^{31}$ Interne Bespr. der Ministerpräsidenten der amerikanischen Besatzungszone mit General Clay v. 1.7. 1947, in: Akten zur Vorgeschichte der Bundesrepublik Deutschland 1945-1949 (künftig AVBRD), Bd. 3: Juni-Dez. 1947, bearb. v. Günter Plum, München/Wien 1982, S. 202-207, hier S. 206. 
und SPD in der Bewertung der amerikanischen Europapolitik zu diesem frühen Zeitpunkt noch recht groß. Kommunistische Angriffe gegen den "Dollarimperialismus" wies der SPD-Vorsitzende Kurt Schumacher mit einer Entschiedenheit zurück, die von Unionspolitikern kaum zu übertreffen war. Die möglichen Auswirkungen des Marshallplans beispielsweise auf die sozialdemokratischen Sozialisierungspläne spielte er herunter, durch das positive „kapitalistische Echo“, die „Zustimmung deutscher Interessenten eines gewissen Kalibers", ließ er sich nicht irritieren. ${ }^{32}$ Wichtiger waren zunächst eine angemessene Beteiligung an der Aufstellung des Plans und die prinzipielle Bereitschaft der Partner, das deutsche Interesse am Export von Fertigwaren anzuerkennen. Unterschiede deuteten sich allenfalls in taktischen Fragen an: Der Anspruch auf Gleichberechtigung als Voraussetzung, nicht erst als Ergebnis deutscher Mitarbeit am europäischen Wiederaufbau, der mit dafür verantwortlich war, daß die SPD bald in eine gewisse auBenpolitische Isolierung geriet, wurde von der CDU nicht mit gleichem Nachdruck erhoben. Die allenfalls in Umrissen erkennbaren amerikanischen Absichten schlossen detaillierte Stellungnahmen, die vermutlich noch andere Differenzen zum Vorschein gebracht hätten, vorerst aus. Entscheidend war für Konrad Adenauer und Kurt Schumacher im Sommer 1947 der erhoffte psychologische Effekt. Der spätere Kanzler sprach, noch unsicher, im Zusammenhang mit dem Marshallplan von „Anzeichen einer Besserung ". ${ }^{33}$ Schumacher zog im Oktober, schon zuversichtlicher, eine erste positive Zwischenbilanz: Die Marshall-Initiative habe „Reserven des Lebenswillens und des Vertrauens" reaktiviert; im Bewußtsein der Deutschen sei „praktisch und seelisch eine neue Situation geschaffen worden". 34

Dieser wachsende Optimismus zeigt sich auch in der wohl ersten längeren Erwiderung auf den amerikanischen Vorschlag aus Kreisen der deutschen Industrie. Bereits am 9. Juni, vier Tage nach der Harvard-Rede, hatte der Hauptgeschäftsführer der Wirtschaftsvereinigung Eisen- und Stahlindustrie, Wilhelm Salewski, eine Denkschrift fertiggestellt, in der er versuchte, die wichtigsten Gedanken Marshalls mit den Interessen der Montanindustrie an Rhein und Ruhr zu verknüpfen. ${ }^{35}$ Bis dahin hatten sich die Überlegungen von Unternehmern und Verbandsfunktionären zur Rekonstruktion „ihrer“ Industrien, zumindest wenn solche Pläne alliierten oder deutschen Stellen zugänglich gemacht werden sollten, meist auf die jeweilige Zone konzentriert, seltener auf alle vier zusammen. Diese in kluger Voraussicht geübte „Bescheidenheit“ wurde nun langsam zugunsten offensiverer Pläne aufgegeben, die auch die Nachbarländer einschlossen; Salewskis interne Denkschrift ist für diesen Wandel ein früher, deutlicher Beweis. Ohne

\footnotetext{
32 Vgl. insbesondere Schumachers Grundsatzreferat auf dem Nürnberger Parteitag der SPD v. 20. 6. 1947, in: Kurt Schumacher, Reden - Schriften - Korrespondenz 1945-1952, hrsg. v. Willy Albrecht, Berlin/ Bonn 1985, S. 488-491. Er sagte dort u. a.: „Ein Projekt von so gewaltigen Ausmaßen ist niemals ein Geschäftsprojekt. Ein Projekt von solcher Enormität ist stets ein Stück echter Hilfeleistung und Verantwortung vor der Welt und für die Welt." Zur Resonanz in der SPD vgl. Hans-Peter Ehni, Die Reaktion der SPD auf den Marshall-Plan, in: Haberl/Niethammer, Marschall-Plan, S. 217-230; speziell zu Schumacher vgl. Wolfgang Benz, Kurt Schumachers Europakonzeption, in: Herbst/Bührer/Sowade, Marshallplan, S. 47-61.

${ }^{33}$ Adenauer an den österr. Gesandten Wildner v. 23. 7. 1947, in: Adenauer. Briefe 1947-1949, bearb. v. Hans Peter Mensing, Berlin 1984, S. 35.

${ }^{34}$ Rede Schumachers auf dem Jahreskongreß der American Federation of Labor v. 14. 10. 1947, in: Schumacher, Reden, S. 562-569, hier S. 565.

35 Vgl. zum folgenden Werner Bührer, Ruhrstahl und Europa. Die Wirtschaftsvereinigung Eisen- und Stahlindustrie und die Anfänge der europäischen Integration 1945-1952, München 1986, S. 85-88.
} 
über die möglichen Absichten der Regierung in Washington zu spekulieren, griff er einen ihm zentral erscheinenden Aspekt der Rede des amerikanischen Außenministers heraus, nämlich die Forderung nach einer gemeinsamen Initiative und gemeinsamen Anstrengungen der eưopäischen Länder. Ansatzpunkte für eine solche Zusammenarbeit erblickte er vor allem in den Kooperationsformen und -erfahrungen der Zwischenkriegszeit, namentlich eine Wiederbelebung der internationalen Kartelle erschien ihm, trotz zu erwartender Widerstände, unbedingt geboten. Auf die Rolle der Deutschen in einem solchen Kartell wie im Rahmen des geplanten Hilfsprogramms überhaupt ging Salewski nicht eigens ein, doch lassen Tenor und Tendenz seiner Überlegungen keinen Zweifel daran, daß ihm ein gleichberechtigter Status vorschwebte. Gewiß bemühte er sich später, als die Konturen des „European Recovery Program“ deutlicher sichtbar waren, um eine bessere Abstimmung der Verbands- und Brancheninteressen mit diesem Programm. Dennoch war dieser frühe Versuch, im Windschatten der amerikanischen Europapolitik eigene, "nationale“, branchen- oder firmenspezifische Zwecke zu verfolgen, nicht untypisch für die künftige deutsche Integrationspolitik. Zwar ließen sich, dies zählt mittlerweile zu den Binsenweisheiten der Integrationsforschung, auch die übrigen Länder von ihren "nationalegoistischen" Zielen leiten, doch waren sie nicht in gleichem Maße wie die Deutschen gezwungen, ihre Interessen in „europäischer Übersetzung “ zu artikulieren.

Die Chancen, die Marshalls Offerte in sich barg, wurden also in den maßgeblichen politischen und wirtschaftlichen Kreisen Westdeutschlands sehr wohl erkannt oder doch zumindest erahnt: Noch vorhandene restriktive Elemente der alliierten Besatzungspolitik konnten nun leichter attackiert, Demontagen und Entflechtung als unvereinbar mit dem ERP angeprangert werden. Die Forderung nach Wiederankurbelung der Wirtschaft und des Außenhandels bedurfte kaum noch einer Begründung. Von der Beteiligung deutscher Experten an der Ausarbeitung und Abwicklung des Programms, mit der schon aus Effizienzgründen fest gerechnet wurde, erhoffte man sich, erstmals nach Kriegsende, wieder die Möglichkeit, auf die wirtschaftliche Entwicklung in Europa aktiv Einfluß zu nehmen. Auf diesem Wege war es wohl auch am besten und vor allem am schnellsten möglich, die außenpolitische Isolierung zu überwinden und die Rehabilitierung Deutschlands voranzubringen, wenngleich sich gerade ehemalige Diplomaten anfangs schwer taten, diesen Aspekt des Wiederaufbauprojekts wahrzunehmen. ${ }^{36}$

Fraglich ist hingegen, ob zu diesem frühen Zeitpunkt die Implikationen oder gar, je nach Standpunkt, Gefahren des Marshallplans ebenso hellsichtig begriffen wurden. Mit Blick auf seine mutmaßlichen Konsequenzen für die Wirtschaftsordnung ist dies im Falle der SPD nachdrücklich bezweifelt worden. ${ }^{37}$ Freilich war die Hoffnung sozialdemokratischer Politiker, trotz des Marshallplans eigene Konzepte verwirklichen zu können, nicht so illusionär, wie es im Rückblick scheinen mag. Zum einen wies das spätere ERP, wie bürgerlich-liberale Kräfte in den Westzonen bald monierten, selbst planerische Züge auf; zum anderen war das Spektrum der Wirtschaftsformen, die aus amerikani-

\footnotetext{
36 Vgl. Eugen Budde, Gibt es noch eine deutsche Außenpolitik? Betrachtungen zur Politik und Diplomatie cines geschlagenen Staates, Stuttgart 1947, der mit seinen Überlegungen noch ganz in den Bahnen herkömmlichen außenpolitischen Denkens blieb.

${ }^{37}$ Vgl. Ehni, Reaktion, S. 219.
} 
scher Sicht noch akzeptabel waren, doch größer, als die deutsche Entwicklung zunächst vermuten läßt. Beispielsweise waren „britische Verhältnisse“ in Westdeutschland im Sommer 1947 nicht von vornherein ausgeschlossen, und sehr viel mehr, etwa eine generelle Abschaffung des Privateigentums an den Produktionsmitteln, verlangte in der SPD ohnehin kaum einer der maßgeblichen Politiker. Die Einstellung der Partei zur Initiative des amerikanischen Außenministers glich also damals noch jener der Gewerkschaften, die politische Auflagen einkalkulierten, aber, angesichts der kritischen Ernährungs- und Wirtschaftslage, die Mitarbeit nicht verweigern wollten. ${ }^{38}$ Auch dafür, daß sich die führenden Kräfte in der CDU und in der SPD Illusionen über die Auswirkungen des Marshallplans auf die deutsche Einheit gemacht hätten, gibt es wenig Anhaltspunkte. Spätestens mit Molotows "Nein" in Paris war deutlich geworden, daß die Einbeziehung Westdeutschlands die Spaltung vertiefen würde. Beide Parteien - von Ausnahmen wie Jakob Kaiser in der Ost-CDU abgesehen - reagierten anfänglich nahezu gleich, indem sie auf die Anziehungskraft wirtschaftlich blühender Westzonen verwiesen und die Westintegration kurzerhand zur Voraussetzung einer Wiedervereinigung erklärten. ${ }^{39}$ Dahinter steckte jedoch eine klare Entscheidung: Priorität hatten der Wiederaufbau und, da dieses Ziel nur im westlichen Verbund realisierbar erschien, die westeuropäische $\mathrm{Zu}$ sammenarbeit. Die Implikationen dieser Zusammenarbeit, die in politischen und wirtschaftlichen Kreisen gleichermaßen auf lebhafte Zustimmung stieß, waren den Deutschen vermutlich am wenigsten bewußt. Die Beteiligung am Marshallplan bedeutete nämlich den Einstieg in die - von manchen nationalkonservativen Politikern verächtlich so genannte-„multilaterale deutsche Kongreßpolitik“. Die Konsequenzen einer solchen „vernetzten“ Außenpolitik für den eigenen Handlungsspielraum, ihr „Zähmungspotential“, machten sich wohl die wenigsten jener begeisterten deutschen „Europäer der ersten Stunde" klar - möglicherweise in der richtigen Erkenntnis, daß es eine Alternative zu Bedingungen, die für die Mehrheit der "politischen Klasse“ akzeptabel gewesen wären, ohnehin nicht gab. ${ }^{40}$ Ein zumindest vorsichtiger Optimismus zeichnete öffentliche und private Stellungnahmen aus, von einer neuerdings wieder öfters behaupteten Zerknirschtheit oder gar Unterwürfigkeit war nichts zu spüren. Im Gegenteil: Die Selbstverständlichkeit, ja manchmal fast schon Dreistigkeit, mit der manche Politiker und

${ }^{38}$ Vgl. Ulrich Borsdorf, In Kauf genommen. Der Marshall-Plan und die Zweiteilung der Einheitsgewerkschaft in Deutschland, in: Haberl/Niethammer, Marshall-Plan, S. 194-211. Wenn der Vorsitzende des Gewerkschaftsbundes der britischen Zone, Hans Böckler, Mitte 1948 dazu aufrief, „die Vorteile restlos auszuschöpfen und all dem rechtzeitig zu begegnen, was (die Gewerkschaften) ... als Nachteile des Plans" empfänden, beschrieb er recht genau den Ansatz auch der SPD; ebd., S. 208. Vgl. auch ErnstDieter Köpper, Gewerkschaften und Außenpolitik. Die Stellung der westdeutschen Gewerkschaften zur wirtschaftlichen und militärischen Integration der Bundesrepublik in die Europäische Gemeinschaft und in die NATO, Frankfurt a. M./New York 1982, bes. S. 32-42.

${ }^{39}$ Für die SPD vgl. Ehni, Reaktion, S. 220f.; grundsätzlich Kurt Thomas Schmitz, Deutsche Einheit und Europäische Integration. Der sozialdemokratische Beitrag zur Außenpolitik der Bundesrepublik Deutschland unter besonderer Berücksichtigung des programmatischen Wandels einer Oppositionspartei, Bonn 1978. Für die CDU Hans-Peter Schwarz, Vom Reich zur Bundesrepublik, Deutschland im Widerstreit der außenpolitischen Konzeptionen in den Jahren der Besatzungsherrschaft 1945-1949, 2. erw. Auflage Stuttgart 1980, bes. S. 326-330 und 478 f.; Josef Foschepoth, Westintegration statt Wiedervereinigung: Adenauers Deutschlandpolitik 1949-1955, in: ders. (Hrsg.), Adenauer und die Deutsche Frage, Göttingen 1988, S. 29-60; Horstwalter Heitzer, Die CDU in der britischen Zone 1945-1949. Gründung, Organisation, Programm und Politik, Düsseldorf 1988, bes. S. 563-576.

40 Vgl. Schwarz, Die gezähmten Deutschen, bes. S. 40-42. Das „Kongreßpolitik“-Zitat stammt von Franz Josef Strauß. 
Industrielle, offenbar mit jenem unter Deutschen verbreiteten „notorisch guten Gewissen“ ausgestattet, deutsche "Rechte“ glaubten einfordern zu können, war - zwei Jahre nach Kriegsende - recht bemerkenswert. Etwas mehr Sensibilität bewies da schon Konrad Adenauer: „Wäre es nicht klug, wäre es nicht wahrhaft groß und edelmütig, wenn Frankreich jetzt dem am Boden liegenden Deutschland die hilfreiche Hand, die sich von Amerika aus entgegenstreckt, nicht beiseite schöbe?" 41 Mit diesem Appell an den „Erbfeind" bewies er nicht nur ein sicheres Gespür für den angemessenen Ton, sondern auch für die wichtigste Hürde, die es auf dem Weg zur Gleichberechtigung zu überwinden galt.

\section{Westdeutschland in den CEEC-Planungen}

Am 12. Juli 1947 versammelten sich die Vertreter von sechzehn europäischen Staaten in Paris, um über das amerikanische Angebot zu beraten. Der Einladung, die der britische Außenminister Bevin und sein französischer Kollege Bidault unmittelbar nach dem Scheitern der Dreierkonferenz mit dem sowjetischen Außenminister Molotow an alle europäischen Regierungen mit Ausnahme der spanischen geschickt hatten, leisteten somit nur diejenigen Folge, die sich dem „Westen“ zurechneten; die Westzonen und Triest waren in Paris noch nicht vertreten. Der Brite Sir Oliver Franks wurde zum Präsidenten des "Committee of European Economic Cooperation“ gewählt. Die beiden anderen wichtigen Ämter erhielten Franzosen zugesprochen: als Generalberichterstatter fungierte Hervé Alphand, der Leiter der Wirtschaftsabteilung des Quai d' Orsay, als Generalsekretär Claude Serreulles.

Auch wenn im State Department im Sommer 1947 kein detaillierter Plan vorlag, hatten sich in der inneramerikanischen Diskussion doch einige Grundsätze herauskristallisiert, über die zumindest die Regierungen Frankreichs und Großbritanniens vor den $\mathrm{Pa}$ riser Beratungen unterrichtet wurden. Dazu zählte, neben dem amerikanischen Interesse an einem gemeinsamen europäischen Vorschlag und einer zeitlichen Befristung des Hilfsprogramms, auch die Teilnahme der Westzonen. ${ }^{42}$ Wie sich die verantwortlichen Stellen in Washington eine deutsche Beteiligung im einzelnen vorstellten, ging aus einer gemeinsamen Instruktion der Minister Marshall, Patterson und Forrestal an die US-Militärregierung vom 11. Juli 1947 hervor: „The US should make known its willingness“, hieß es beispielsweise, "to have its zone of Germany collaborate fully on the preparation of European proposals to reduce the European requirement for aid from the US ... No initiative on the part of the US zone ... is called for, but the occupied area must be represented when European recovery plans are being prepared." Weiter sollte die Militärregierung darauf achten, daß Produktion und Export auf die Bedürfnisse der übrigen europäischen Länder zugeschnitten waren; Bedingung war freilich, daß diese Länder dafür in den Westzonen benötigte Waren lieferten oder Devisen bereitstellten. Für den Fall, daß bestimmte übergreifende „europäische“ Ziele nur zu Lasten der Westzonen zu

\footnotetext{
${ }^{41}$ Rede auf dem ersten Parteitag der CDU der britischen Zone v. 14.-15. 8. 1947, abgedruckt in: Konrad Adenauer und die CDU der britischen Besatzungszone 1946-1949. Dokumente zur Gründungsgeschichte der CDU Deutschlands, eingel. u. bearb. v. Helmuth Pütz, Bonn 1975, S. 330-351, hier S. 350. ${ }^{42}$ Vgl. van der Beugel, Marshall Aid, S. 63-66.
} 
erreichen waren, stellten die drei Minister entsprechende Mittel in Aussicht, um jegliche Verzögerung beim Aufbau einer lebensfähigen deutschen Wirtschaft zu vermeiden. Westeuropa und Westdeutschland sollten also gleichzeitig wiederaufgebaut werden. ${ }^{43}$ Angesichts dieser beabsichtigten Parallelisierung der Rekonstruktionsbemühungen von einem „German-based European recovery program" zu sprechen, das „die“ Amerikaner im Sinn gehabt hätten ${ }^{44}$, erscheint eher abwegig. Dessen ungeachtet war damit zu rechnen, daß in Frankreich schon die geplante Gleichbehandlung des vormaligen Kriegspartners, kaum drei Jahre nach der Befreiung des Landes, auf heftigen Widerstand stoßen würde.

\section{a) Der revidierte Industrieniveauplan}

Der vom Alliierten Kontrollrat im März 1946 verabschiedete, für alle vier Zonen gültige erste „Plan für Reparationen und den Nachkriegsstand der deutschen Wirtschaft" war noch ganz von der Absicht der Siegermächte geprägt gewesen, dem in Europa weitverbreiteten Sicherheitsverlangen durch eine Reduzierung des deutschen Industriepotentials Genüge zu tun und die „überschüssigen“, zur Demontage vorgesehenen Quantitäten festzulegen. Produktionsverbote und -beschränkungen sollten sicherstellen, daß die Erzeugung 50 bis 55 Prozent des Standes von 1938 nicht überschritt. Die Ein- und Ausfuhren sollten sich im Jahre 1949 auf jeweils 3 Milliarden Mark belaufen; dies entsprach 55 bzw. 57 Prozent des Wertes von 1938, als das Außenhandelsaufkommen freilich um mehr als die Hälfte unter dem Niveau der späten zwanziger Jahre lag, oder 21 bzw. 25 Prozent des Wertes von 1928.45 Selbst von solchen niedrigen Ziffern war die reale Produktions- und Außenhandelsentwicklung jedoch noch weit entfernt.

Diese Einsicht, zusammen mit dem frischen Eindruck der Krise des Winters 1946/47 und dem Wunsch, die zum 1. Januar 1947 gegründete Bizone durch eine Verbesserung der wirtschaftlichen Lage zu stabilisieren, ließ auf seiten der beiden Militärgouverneure den Entschluß zur Revision des alten Industrieplans reifen, noch ehe sie während der Moskauer Außenministerkonferenz im Frühjahr 1947 von Marshall und Bevin den offiziellen Auftrag dazu erhielten. ${ }^{46}$ Ohne die Reparationsforderungen und das Sicherheitsbedürfnis der vom Deutschen Reich angegriffenen und besetzten Länder außer acht zu lassen, richteten Clay und sein britischer Kollege, Sir Brian Robertson, ihr Hauptaugenmerk darauf, die notwendigen Voraussetzungen zu schaffen, die es der Bizone ermöglichen sollten, wirtschaftlich „auf eigenen Füßen“ zu stehen. Am 12. Juli meldete Clay den erfolgreichen Abschluß der Arbeiten nach Washington; gleichzeitig kündigte er für

\footnotetext{
+3 Adjudant General, War Dept., an OMGUS v. 11.7. 1947, IfZA, MF 260, POLAD/806-5. Vgl. auch Gimbel, Origins, S. 224.

*4 So Gimbel, Origins, S. 255.

${ }^{45}$ Vgl. aus zeitgenössischer amerikanischer Sicht Benjamin U. Ratchford/William D. Ross, Berlin Reparations Assignment. Round One of the German Peace Settlement, Chapel Hill 1947. Neue Darstellungen finden sich bei Krieger, Clay, S. 133-139, u. Mai, Kontrollrat, S. 312-327. In deutscher Übersetzung ist der Plan abgedruckt in Reparationen, Sozialprodukt, Lebensstandard. Versuch ciner Wirtschaftsbilanz, Bremen 1947, S. 93-96. Die Vergleichszahlen für 1938 und 1928 nach Dietmar Petzina, Die deutsche Wirtschaft in der Zwischenkriegszeit, Wiesbaden 1977, S. 123.

t6 Vgl. Gimbel, Origins, S. 220 f. Zur Winterkrise und zur Bizonengründung vgl. Werner Abelshauser, Wirtschaft in Westdeutschland 1945-1948. Rekonstruktion und Wachstumsbedingungen in der amerikanischen und britischen Zone, Stuttgart 1975, S. 94-100. Dic einsetzende Diskussion über die Revision ist dokumentiert in Clay Papers, I, S. 332-335, 338-340; FRUS 1947, II, S. 472-475.
} 
den 16. Juli die Veröffentlichung des neuen, bizonalen Plans an. Erste spärliche Informationen über den Inhalt und vor allem der vorgesehene Zeitpunkt für die öffentliche Bekanntgabe der Vereinbarung zwischen dem amerikanischen und dem britischen Militärgouverneur lösten in Frankreich jedoch heftige Proteste aus. Die französische Regierung wertete diese anglo-amerikanische Initiative nämlich als Versuch, die am 12. Juli in Paris eröffnete Konferenz über den Marshallplan in der Frage der Behandlung Westdeutschlands zu präjudizieren. In einem dramatischen, durch eine Rücktrittsdrohung noch verstärkten Appell an Außenminister Marshall vom 17. Juli bot Bidault seine ganze Überzeugungskraft auf, um die Verabschiedung des revidierten Industrieniveauplans zu stoppen. Angesichts der großen Lücke zwischen tatsächlicher und erlaubter Produktion erschien ihm eine neue, verpflichtende Abmachung über die wirtschaftliche Zukunft des besetzten Landes keineswegs als drängende Aufgabe. Frankreich wolle Deutschland weder aushungern, noch seine Ressourcen brachliegen lassen: „But if on the morrow of the Paris Conference, at a time when we are still filled with uncertainty regarding ourselves, there is a certainty for Germany, the consequences of this priority cannot be escaped. " 47 Dieser massiven Intervention hätte es freilich gar nicht mehr bedurft, denn das amerikanische Außenministerium hatte, in Abstimmung mit dem Kriegsministerium und dem britischen Außenminister Bevin, bereits zwei Tage zuvor Clay angewiesen, die Veröffentlichung des Plans zu verschieben. ${ }^{48}$ Ein möglichst ungestörter und erfolgreicher Verlauf der Pariser Konferenz, die für das Schicksal des Marshallplans zweifellos von entscheidender Bedeutung war, erschien dem State Department wichtiger als der psychologische Effekt einer Stimulierung des Aufbauwillens in der Bizone, den sich der amerikanische Militärgouverneur von der Anhebung der Produktionsobergrenzen in einigen wichtigen Industriezweigen versprach. Die Rücksichtnahme auf die französische Regierung ging allerdings nicht so weit, daß den Experten des Quai d'Orsay die Gelegenheit gegeben worden wäre, den Industrieniveauplan nach ihren Vorstellungen tatsächlich zu verändern; die zahlreichen zwei- und dreiseitigen Besprechungen, die im Juli und $\mathrm{Au}$ gust 1947 stattfanden, dienten aus anglo-amerikanischer Sicht eher dazu, die französischen Befürchtungen zu zerstreuen und dadurch die Aufregung zu dämpfen. Einschneidende Korrekturen konnten schließlich schon mit dem Hinweis abgeblockt werden, daß die Vorgaben lediglich für die Bizone gelten sollten. Der am 29. August endlich veröffentlichte „Revidierte Plan für das Industrieniveau der britischen und amerikanischen Zone Deutschlands“ wies jedenfalls keine gravierenden Veränderungen gegenüber dem Stand vom Juli auf. ${ }^{49}$

Dennoch enthielt der Plan nur wenige Punkte, welche die Aufregung in Paris wirklich gerechtfertigt erscheinen ließen. Begründet wurde die Revision in der Präambel damit, daß sich weder die Bizone noch Deutschland als Ganzes wirtschaftlich erholen könnten, wenn die alten Vorschriften in Kraft blieben - von dem unentbehrlichen Beitrag zur

\footnotetext{
${ }^{47}$ Bidault an Marshall v. 17. 7. 1947, FRUS 1947, II, S. 991 f.; eine Botschaft gleichen Inhalts erhielt auch der britische Außenminister Bevin. Vgl. auch Milward, Reconstruction, S. $71 \mathrm{f}$.

${ }^{48}$ Marshall an amerikan. Botschaft in Frankreich v. 15.7. 1947, FRUS 1947, II, S. 987 f. Zur Reaktion Clays vgl. Clay an Petersen v. 16. 7. 1947, Clay Papers, I, S. 383 f.

${ }^{49}$ Vgl. hierzu Krieger, Clay, S. 282-289; Mai, Kontrollrat, S. 436-449. Die amerikanisch-französischen Unterredungen sind dokumentiert in FRUS 1947, II, S. 983-1049. Die am 29. August veröffentlichte Fassung des „Revised Plan“ findet sich in IfZA, MF 260, 3/406-1/5. Eine deutsche Übersetzung ist abgedruckt in Reparationen, S. 97-100.
} 
Wiedergesundung Europas ganz zu schweigen. Eine Bevorzugung Westdeutschlands, wie von Bidault befürchtet, ließ sich daraus ebensowenig ablesen wie aus den neuen Richtzahlen für die einzelnen Industriezweige. Obwohl die erlaubte Kapazität in der Bizone im Durchschnitt dem Niveau des Jahres 1936 entsprach, lagen - auch für den Export - wichtige Branchen wie die Stahlindustrie oder der Maschinenbau deutlich darunter:

Tabelle 1: Stahlindustrie und Maschinenbau der Bizone im revidierten Industrieniveauplan

$\begin{array}{lccc} & \begin{array}{c}\text { geschätzte } \\ \text { Produktion } \\ 1936^{2}\end{array} & \begin{array}{c}\text { zugelassene } \\ \text { Höchstproduktion } \\ \text { gem. rev. Plan }\end{array} & \begin{array}{c}\text { in Prozent } \\ \text { von } 1936\end{array} \\ \begin{array}{l}\text { Stahlindustrie (in Mill. t) } \\ \text { Maschinenbau (in Mill. RM) }\end{array} & 14,9 & 10,7 & 72 \\ \text { Leichte Maschinen } & & & \\ \text { Schwere Maschinen } & 619 & 500^{3} & 80 \\ \text { Werkzeugmaschinen } & 769 & 916^{3} & 119 \\ \text { Quelle: Reparationen, S. 97 } & 206 & 170^{3} & 83 \\ \text { 1) Dazu zählten u. a. Textilmaschinen, holzbearbeitende Maschinen sowie Ausrüstungen für die } \\ \text { Nahrungsmittelindustrie und zur Herstellung von Gebrauchsartikeln. } \\ \text { 2) Gebiet der späteren Bizone. }\end{array}$

Gleichwohl gab die Planziffer für die Stahlproduktion noch am ehesten Anlaß zur Besorgnis. Zwar machte die zugelassene Produktion nur etwas mehr als die Hälfte der vorhandenen Kapazität aus, so daß die französische Stahlindustrie, wie im Monnetplan vorgesehen, durch eine Erweiterung ihrer Kapazitäten einen beträchtlichen Teil der deutschen Produktion hätte übernehmen können; die Realisierung dieses Vorhabens hing aber ganz entscheidend von einer hohen und kontinuierlichen Kohlenzufuhr aus dem Ruhrgebiet ab, die bei einer bizonalen Erzeugung von fast 11 Mill. Tonnen pro Jahr keineswegs als gesichert angesehen werden konnte. Gerade in der Frage der Stahlquote zeigten sich Amerikaner und Briten jedoch unnachgiebig - freilich nicht, um den Monnetplan zu torpedieren, sondern weil das Ziel der "selbsttragenden Wirtschaft" auf dem Spiel stand. Auf dieses Ziel waren auch die neuen Obergrenzen für die übrigen eingeschränkten Industriezweige - Nichteisen-Metalle, Kraftfahrzeugindustrie, Feinmechanik und Optik, Elektroindustrie, Chemie und Kunststoffindustrie - zugeschnitten. Die Notwendigkeit, Erzeugnisse der Metallindustrie, des Maschinenbaus und der Chemischen Industrie zu exportieren, wurde ausdrücklich anerkannt, mehr noch: Wegen des höheren Einfuhrbedarfs an Lebensmitteln sollte die Ausfuhr dieser und der anderen beschränkten Industrien sogar "größer als vor dem Kriege sein. " ${ }^{50}$ Der amerikanisch-britische Plan nahm mithin Kurs auf eine partielle Restauration der früheren Rolle der deutschen Wirtschaft im arbeitsteiligen Geflecht der europäischen Ökonomie, ohne indes die Bemühungen um den deutschen Wiederaufbau mit den europäischen Erfordernissen abzustimmen. Er war auf die Bizone ausgerichtet, doch nicht in einer Weise, welche die

${ }^{50}$ Revidierter Industrieniveauplan, zit. n. Reparationen, S. 98. 
französischen Proteste gegen die vermeintliche Bevorzugung des einstigen Kriegsgegners berechtigt erscheinen ließe.

Den Betroffenen selbst reichte die amerikanisch-britische Initiative hingegen bei weitem nicht aus. Während es der bizonale Wirtschaftsrat noch bei einer recht zahmen Erklärung beließ und lediglich die Frage stellte, ob „das Ziel der wirtschaftlichen Gesundung Deutschlands im Rahmen eines wiedergesundenden Europas unter den Begrenzungen, die auch der revidierte Industrieplan noch vorsieht, erreicht werden " könne ${ }^{51}$, waren die Stimmen aus der Industrie bzw. aus ihr nahestehenden Kreisen schon deutlicher. Die Wirtschaftsvereinigung Eisen- und Stahlindustrie wandte sich vor allem gegen die ihrer Ansicht nach zu hohe Schätzung der verbliebenen Rohstahlkapazität: Statt 19,2 Mill. Tonnen im Jahr errechnete der Verband nur 13,7 Mill. Tonnen. Abzüglich der angekündigten Demontagen in Höhe von 3,8 Mill. Tonnen hätte die verbleibende Kapazität demnach nicht einmal mehr den Richtwert des neuen Industrieplans - 10,7 Mill. Tonnen pro Jahr - erreicht. ${ }^{52}$ Ähnlich argumentierte auch die Arbeitsgemeinschaft der Verbände Deutscher Maschinenbauanstalten, die sich im Oktober mit einer umfangreichen Stellungnahme zu Wort meldete. Sie machte zum einen geltend, daß exakte Kapazitätsberechnungen in der Maschinenindustrie grundsätzlich kaum möglich seien; im deutschen Fall komme zum anderen noch erschwerend hinzu, daß das Leistungsvermögen der Fabriken durch die Überbeanspruchung während des Krieges und die geringere Arbeitsproduktivität nachgelassen habe. Kurzum: die "Ausgangskapazitäten“ seien „zu hoch angesetzt“. Noch gravierender war aber der Einwand, der sich gegen die „Unausgeglichenheit zwischen zugelassener Maschinenproduktion und übriger Industrieproduktion" richtete. Trotz aller optischen Verbesserungen würden aufgrund der Bedeutung des Maschinenbaus „praktisch auch alle übrigen Industrien - ob eingeschränkt oder nicht - auf den für die Maschinenproduktion vorgesehenen Stand festgelegt ${ }^{*}$. Weder genüge der neue Plan den Erfordernissen einer sich selbst tragenden deutschen Friedenswirtschaft, so das Fazit der „Arbeitsgemeinschaft“, noch garantiere er den erwünschten Beitrag zum europäischen Wiederaufbau: „Würde man der deutschen Wirtschaft die notwendige Bewegungsfreiheit zurückgeben, so könnte Europa mit wirkungsvolleren Beiträgen zur Wiederherstellung seines Wohlstandes rechnen, als sie den einzelnen Reparationsgläubigern durch Überlassung demontierter Maschinen zuteil" werde. ${ }^{53}$

Hatten sich diese beiden Stellungnahmen in erster Linie auf die Probleme der jeweiligen Branche konzentriert, so unternahm eine Bremer Arbeitsgruppe unter Leitung von Gustav Wilhelm Harmssen, der dort seit Ende 1946 als Senator für Wirtschaftsforschung und Außenhandel amtierte, erstmals den Versuch einer umfassenden Bewertung der Implikationen und Konsequenzen des revidierten Plans. Obwohl es sich dabei um eine im Auftrag der Ministerpräsidenten der Bizone verfaßte und damit zumindest offiziöse Studie handelte, nahmen Harmssen und seine Mitarbeiter kein Blatt vor den

5t Erklärung des Wirtschaftsrates zum Industrieplan, angenommen auf der 4. Vollversammlung am 4./5. 9. 1947, in: Wörtliche Berichte, Bd. 4: Drucksachen, S. 57.

52 Wirtschaftsvereinigung an Wirtschaftsminister Nordrhein-Westfalen v. 28. 10. 1947, Handakten Salewski (künftig HAS; Kopie im Besitz d. Verf.).

${ }^{53}$ Der Maschinenbau im revidierten Industrieplan, Ausarbeitung v. 13. 10. 1947, Bundesarchiv, Koblenz (künftig BA), Z 35, 411, Bl. 61-77. 
Mund. ${ }^{54}$ Zwar räumten sie ein, daß der revidierte Plan „eine tiefere Einsicht in die Notwendigkeiten einer Wirtschaft“ zeigte, die "ohne Hilfe von außen nicht bestehen“ könne, doch die Kommentare zu den Kapazitätsberechnungen und Produktionsvorgaben für die verschiedenen Industriezweige fielen fast durchweg negativ aus. Wichtige Produktionsmittelindustrien wie die Stahlerzeugung oder der Schwer- und Werkzeugmaschinenbau waren nach Ansicht der Bremer Experten "weit unter dem Stand von 1936 quotiert." ${ }^{55}$ Aufgrund anderer Berechnungsmethoden -Amerikaner und Briten legten die „technische Bestandskapazität" zugrunde, während die deutschen Kritiker die „wirtschaftliche Leistungskapazität“ zum Maßstab nahmen - kamen Harmssen und seine Mitautoren zu deutlich niedrigeren Ziffern: Nicht den Stand des Jahres 1936, wie der neue Plan vorgab, sondern „höchstens“ 70 bis 75 Prozent der damals erzeugten Gütermenge hielten sie für erreichtbar. Stelle man dann noch eine im Jahr 1952 um etwa 25 Prozent höhere Bevölkerungszahl in Rechnung, sinke die Pro-Kopf-Produktion sogar auf 60 bis 55 Prozent des Ergebnisses von 1936.56 Noch schärfer ging das Bremer Team mit den anglo-amerikanischen Außenhandelsplanungen ins Gericht. Dem deutschen Handel seien nämlich gerade dort „Barrieren“ errichtet worden, „wo er mit Aussicht auf Erfolg am wirksamsten zu der Besserung der deutschen Wirtschaftslage“ hätte einsetzen können: Schiffe, Flugzeuge, Kraftfahrzeuge, Eisenwaren, Maschinen und Werkzeugmaschinen sowie elektrotechnische Artikel - alles gefragte und konkurrenzfähige Güter durften ja laut Industrieplan überhaupt nicht oder nur in begrenztem Maße produziert werden. Der Plan sei eben „mit unerbittlicher Konsequenz auf das Sicherheitsbedürfnis der Alliierten, hinsichtlich der Ausfuhr aber ohne Zweifel auch auf die Wünsche der alliierten Exportkonkurrenz abgestellt", waren Harmssen und seine Mitarbeiter überzeugt. Anstelle der im revidierten Plan veranschlagten Ein- und Ausfuhren im Wert von jeweils etwa 2,8 Mrd. RM forderten sie deshalb für das „Reich“ Einfuhren in Höhe von 6 und Ausfuhren in Höhe von 7,5 Mrd. RM, weil anders der erlaubte mittlere europäische Lebensstandard für die deutsche Bevölkerung und eine ohne fremde Hilfe existenzfähige Wirtschaft unerreichbare Ziele bleiben würden. ${ }^{57}$

Die Bremer Studie konnte sich des Beifalls der maßgeblichen politischen und wirtschaftlichen Kreise in den Westzonen gewiß sein, mochte der teilweise aggressive Ton auch vielen deutschen Politikern und Behördenchefs überzogen oder zumindest taktisch ungeschickt erschienen sein. Sie machte auf einige Ungereimtheiten und Widersprüche des revidierten Industrieniveauplans aufmerksam, insbesondere auf das Mißverhältnis zwischen den Kapazitäts- oder Erzeugungsquoten im Produktionsgüterbereich und den kaum oder gar nicht eingeschränkten übrigen Industriezweigen. So stichhaltig dieser Einwand auch war, so wenig traf er jedoch auf die reale Entwicklung der industriellen Produktion $\mathrm{zu}$, die in den meisten Fällen noch weit von den neuen Obergrenzen entfernt war:

\footnotetext{
${ }^{54}$ Vgl. dazu und zur deutschen Kritik am Tenor der Harmssen-Studie Heribert Piontkowitz. Anfänge westdeutscher Außenpolitik 1946-1949. Das Deutsche Büro für Friedensfragen, Stuttgart 1978, S. 70 f., 152.

${ }_{55}$ Reparationen, S. 32.

${ }^{56}$ Reparationen, Anlage IV: Wirtschaftskontrolle, deutscher Lebensstandard und künftiges Sozialprodukt, S. $26 \mathrm{f}$.

${ }^{57}$ Reparationen, Anlage IX: Der Ein- und Ausfuhrhandel, bes. S. 14-17.
} 
Tabelle 2: Produktion in der Bizone im Oktober 1947, verglichen mit dem Stand von $1936(=100)$

Mehr als zwei Drittel

Mehr als ein Drittel

Weniger als ein Drittel

$\begin{array}{lr}\text { Elektrizitäts- und Gasversorgung } & 105 \\ \text { Kohle } & 73 \\ \text { Elektroindustrie } & 67 \\ \text { Stahl- und Eisenbau } & 66 \\ \text { Glas und Keramik } & 63 \\ \text { Kautschuk } & 58 \\ \text { Holzindustrie } & 50 \\ \text { Petroleum und Kohleprodukte } & 45 \\ \text { Chemische Industrie } & 41 \\ \text { Maschinenbau und optische Industrie } & 41 \\ \text { Papier und Pappe } & 35 \\ \text { Textilindustrie } & 34 \\ \text { NE-Metalle } & 32 \\ \text { Eisen- und Stahlproduktion } & 26 \\ \text { übrige Metallindustrie } & 26 \\ \text { Fahrzeugbau } & 17\end{array}$

Quelle: Abelshauser, Wirtschaft, S. 44.

Ungeachtet seiner zunächst ohnehin nur "theoretischen“ Schwächen war die Revision des Planes freilich ein deutliches Signal der „Entschlossenheit der angelsächsischen Besatzungsmächte, die Rekonstruktion der Wirtschaft ihrer beiden Zonen aktiver als bisher zu fördern". ${ }^{58}$ In dieser grundsätzlichen Frage herrschte also Übereinstimmung mit Politikern und Wirtschaftsexperten aus der Bizone, ebenso über die einzuschlagende Richtung; selbst das Verhältnis zwischen bizonalem und westeuropäischem Wiederaufbau war unstrittig, denn sowohl der Industrieplan als auch die deutschen Kritiker konzentrierten sich letztlich, von wenigen „europäischen“ Formeln eher ornamentalen Charakters abgesehen, auf das westdeutsche Potential. In den Pariser Verhandlungen über den Marshallplan mußte sich zeigen, ob die Deutschen von ihren möglichen künftigen Partnern im europäischen Verbund ein ähnlich großes Wohlwollen und einen ähnlich großen Spielraum zur Entfaltung der eigenen wirtschaftlichen Energien erwarten konnten, wie dies vor allem die Amerikaner zu gewähren bereit waren.

\section{b) Das „deutsche Problem “ in den Beratungen des Committee of European Economic Cooperation}

Am 9. Juli 1947 trafen Bidault und William Clayton vom State Department zusammen, um über Aufgaben und Ziele der unmittelbar bevorstehenden Marshallplankonferenz zu sprechen. „Deutschland“ stand im Mittelpunkt der Gespräche. Der französische Außenminister unterstrich einmal mehr, daß auf keinen Fall der Eindruck entstehen dürfe, als ob der Wiederaufbau des besiegten Landes Priorität genießen und Frankreich auf seine Reparationsforderungen verzichten werde. Andernfalls werde die Konferenz scheitern, mehr noch: „Il n'y aurait pas d'Europe. C Clayton bemühte sich zwar, Bidault davon zu überzeugen, daß niemand in den Vereinigten Staaten die Absicht habe, ein Eu-

58 Abelshauser, Wirtschaft, S. 99. 
ropa mit einem dominanten Deutschland als Mittelpunkt aufzubauen, konnte jedoch nicht alle Zweifel ausräumen. ${ }^{59}$ Es lag ganz auf dieser französischen Linie, daß keine Vertreter der drei westlichen Besatzungszonen nach Paris eingeladen worden waren; Deutsche wären ohnehin nicht in Frage gekommen, doch auch ein Mitglied der amerikanischen Militärregierung mußte aus französischer Sicht aufgrund der deutschlandpolitischen Differenzen noch "gefährlich" genug erscheinen. Verständlicherweise verzichteten die zuständigen Stellen in Frankfurt nach dieser Entscheidung darauf, von sich aus Beobachter in die französische Hauptstadt zu schicken. ${ }^{60}$

Falls die französische Regierung gehofft haben sollte, in Abwesenheit „bizonaler“ Sprecher leichteres Spiel zu haben und die Behandlung des „deutschen Problems“ ausklammern oder wenigstens in ihrem Sinne lenken zu können, wurde sie rasch enttäuscht. Aus ureigenstem Interesse nahmen sich nämlich die Beneluxländer dieses Problems an, das damit von Anfang an zu den beherrschenden Konferenzthemen zählte. ${ }^{61}$ Schon in der ersten Woche prallten die gegensätzlichen Standpunkte aufeinander. Als beispielsweise der niederländische Delegierte für eine vollständige Nutzung der in Europa, insbesondere in Westdeutschland, vorhandenen Kapazitäten plädierte und damit indirekt den Monnetplan in Frage stellte, reagierte Hervé Alphand, der französische Vertreter, recht heftig: "If the Dutch meant that the recovery of Germany's economy had to be pursued at the the same rate as for the rest of Europe, and if it was meant that this approach implied a full utilization of Germany's industrial capacity, the French attitude had to be in the negative. " Lediglich eine Steigerung der Kohlenförderung und der landwirtschaftlichen Erzeugung war der Franzose bereit zuzugestehen, die Entwicklung in anderen Branchen, vor allem in der Stahlindustrie, sah er hingegen in erster Linie unter Sicherheitsgesichtspunkten; ohnehin gehörte die Rekonstruktion der deutschen Wirtschaft nach Alphands Auffassung nicht zu den Aufgaben, welche die Konferenz zu lösen hatte. ${ }^{62}$ Die französische Regierung, so kommentierte der amerikanische Botschafter in Paris, Jefferson Caffery, die erste Woche der CEEC-Beratungen, habe ihren „pastoral approach“ gegenüber Westdeutschland nicht aufgegeben und beharre darauf: „Security lies in ,pulling heavy industrial teeth" of Germany. "63 Dieses Sicherheitsargument überzeugte in Washington jedoch immer weniger. Da die westdeutsche Produktion noch nicht einmal 50 Prozent des Vorkriegsniveaus ausmachte, während in Frankreich der Produktionsstand von 1938 bereits annähernd erreicht war, fiel es gewiß nicht nur Kennan und dem Policy Planning Staff schwer, die französischen Befürchtungen ernst zu nehmen. ${ }^{64}$ Immerhin zeigte die Hartnäckigkeit, mit der die Beneluxgruppe, zunehmend unterstützt von anderen kleineren Ländern, ihren Standpunkt verteidigte, allmählich Wirkung: Die Delegation des Gastgeberlandes willigte Ende Juli ein, daß die Befehlsha-

${ }^{99}$ Besprechung Bidault - Clayton v. 9. 7. 1947, MAE, Y 1944-1949, Vol. 129. Vgl. auch Caffery an Secretary of State v. 11. 7. 1947, FRUS 1947, III, S. 328-330.

60 OMGUS an Adjudant General, War Dept., v. 13. 7. 1947, IfZA MF 260, POLAD/784-2. Ob die britische Militärregierung, wie in diesem Telegramm behauptet, Vertreter nach Paris entsandte, ließ sich nicht definitiv klären. Eine „offizielle“ Vertretung gab es nicht, doch nahmen Angehörige der britischen Militärregierung als Mitglieder der britischen Delegation an den Beratungen teil.

61 Vgl. dazu van der Beugel, Marshall Aid, S. 72-74; Gimbel, Origins, S. 254-266; Milward, Reconstruction, S. 69-89; Hogan, Marshall Plan, S. 60-82.

62 Caffery an State Dept. v. 17. 7. 1947, IfZA, MF 260, POLAD/784-2; Draft record of second meeting CEEC v. 17. 7. 1947, Public Record Office, Kew (künftig PRO), FO 371/62568.

${ }^{63}$ Caffery an Secretary of State v. 20. 7. 1947, FRUS 1947, III. S. 333-335, hier S. 334.

64 Vgl. Memorandum Policy Planning Staff v. 21. 7. 1947, ebenda, S. 335-337. 
ber der Besatzungszonen den gleichen Fragebogen zugestellt bekamen, den auch die übrigen teilnahmewilligen Länder erhalten hatten. Der Industrieplan von 1946 diente also nicht länger „automatisch“ als Richtschnur westdeutscher Bedarfsplanungen im Rahmen des europäischen Wiederaufbauprogramms. ${ }^{65}$

Ungeachtet solcher Anzeichen französischer Kompromißbereitschaft, die auch Caffery registriert hatte, wuchs auf amerikanischer Seite die Neigung, die Rolle des - mehr oder weniger - passiven Beobachters aufzugeben und, getreu der schon von Marshall in seiner Rede gebrauchten Formel "friendly aid“, aktiv in die Pariser Beratungen einzugreifen. Ausschlaggebend dafür war vor allem die Sorge, am Ende könnten die Europäer einen für Regierung und Kongreß inakzeptablen Bericht vorlegen; einer der möglichen kritischen Punkte war, nach wie vor, die Behandlung Westdeutschlands. Wo aus amerikanischer Sicht die Grenze berechtigter Forderungen erreicht war, verdeutlichte Clayton anläßlich eines Treffens mit den Botschaftern Caffery und Douglas, Robert Murphy sowie Paul Nitze vom State Department vom 4. bis 6. August 1947 in Paris, als er zwischen „legitimen“ und „illegitimen“ Interessen Frankreichs unterschied. Zur ersten Kategorie zählte er „militärische Sicherheit“ und den Wunsch, die wirtschaftliche Abhängigkeit von "Deutschland“ zu verringern; "the illegitimate interest was to increase France's competitive position vis-a-vis Germany by the removal of a large part of Germany's competitive potential." Clayton wollte die französische Regierung nicht im unklaren darüber lassen, daß sie bei der Verfolgung „illegitimer“ Interessen nicht mit der Hilfe Washingtons rechnen konnte. ${ }^{66}$ Diskutiert wurde auch die Möglichkeit, unmittelbar nach einer Einigung zwischen den drei Westmächten den revidierenden Industrieplan auf der Marshallplankonferenz zu präsentieren, um den Teilnehmern einen Eindruck zu vermitteln, wie die deutsche Wirtschaft nach amerikanischer Auffassung strukturiert sein sollte. ${ }^{67}$

Daß es zumindest auf der Ebene der Militärregierungen keine Meinungsverschiedenheiten zwischen Briten und Amerikanern gab und der revidierte Plan de facto bereits als Grundlage ihrer Planungen diente, zeigte der „Report on the Bizonal Balance of Payments 1948-1952“ der britischen Control Commission vom 13. August 1947. Dieser Bericht, offensichtlich als Antwort auf den CEEC-Fragenkatalog konzipiert ${ }^{68}$, stützte sich

${ }^{65}$ Vgl. Caffery an Secretary of State v. 29. 7. 1947, ebenda, S. 339-341. Zumindest im Falle der US-Zone wurde der Fragebogen über den amerikanischen Botschafter in Paris an die Militärregierung geleitet; vgl. dazu CEEC-Generalsekretär Serreulles an US-Botschafter v. 1.8. 1947, IfZA, MF 260, POLAD/ 784-3.

${ }^{66}$ Haraldson, Memorandum for record v. 8. 8. 1947, IfZA, MF 260, POLAD/784-3. In der in FRUS 1947, II, S. 345-34 abgedruckten Version des Dokuments fehlt die nähere Charakterisierung der „illegitimen“ Interessen; da dieses Dokument außerdem länger ist, scheint es mindestens zwei Versionen des Memorandums gegeben zu haben.

${ }^{67}$ Memorandum Hickerson v. 11. 8. 1947, FRUS 1947, III, S. 351-355.

${ }^{68}$ Report on the bi-zonal balance of payments 1948-1952, IfZA, MF 260, 11/101-2/7. Das Dokument selbst gibt keinen Aufschluß über Z weck und Adressat. Da jedoch die Überschriften der ersten vier Abschnitte des Reports fast wörtlich mit den entsprechenden Punkten des französischen Fragebogenentwurfs übereinstimmen, können an seinem Verwendungszweck kaum Zweifel bestehen; vgl. Questionnaire drafted by the French Delegation v. 16. 7. 1947, PRO, FO 371/62568. Den offiziellen „Plan for the combined Area submitted to the Committee of European Economic Cooperation, Aug. 1947“, der von OMGUS-Chronisten erwähnt wurde, konnte ich nicht ausfindig machen; vgl. „Production and Export Trade Problems for the Bi-Zone in Mid-1947", IfZA, MF 260, 3/409-1/8-9. Einzelne Daten und Formulierungen in der OMGUS-Darstellung lassen jedoch darauf schließen, daß der „Plan for the combined Area" und der "Report on the bi-zonal balance of payments" über weite Strecken identisch gewesen sein dürften. 
auf die Daten des revidierten Plans. Er betonte beispielsweise die Notwendigkeit umfangreicher Ausfuhren gerade jener Güter, die traditionellerweise etwa zwei Drittel des deutschen Exports ausgemacht hatten, nämlich Stahlprodukte, Maschinen und chemische Erzeugnisse, und verteidigte insbesondere die höhere Stahlquote von etwa 10 Millionen Tonnen als unabdingbare Voraussetzung für die Lebensfähigkeit der westdeutschen Wirtschaft; ein Engpaß in diesem Bereich, so warnten die Verfasser des Reports, werde nicht nur die Produktion im Investitionsgütersektor beeinträchtigen, sondern auch die Landwirtschaft, das Transportwesen und die Konsumgüterindustrien. Hoffnungen auf weitere Beiträge zum Wiederaufbau der übrigen Teilnehmerländer in Form von Kohle, Energie und Holz dämpften die Autoren hingegen mit dem Hinweis auf den internen Bedarf der Bizone. Statt dessen plädierten sie für eine Normalisierung der Handelsbeziehungen durch angemessene Wechselkurse, die Erleichterung von geschäftlichen Auslandskontakten und -reisen sowie den Schutz der deutschen Warenzeichen. Mit diesem Vorschlag befanden sich die Experten der britischen Militärregierung zumindest der Tendenz nach im Einklang mit den Vorstellungen deutscher Stellen, die in diesem Stadium freilich keinerlei Mitspracherecht bei der Formulierung bizonaler Produktions- und Außenhandelsprogramme besaßen, und sogar sehr weitgehend mit jenen der Beneluxländer.

Die Entschlossenheit der Regierungen in Brüssel, Den Haag und Luxemburg, diese erste Gelegenheit, die westalliierte Deutschlandpolitik mehr als nur punktuell zu beeinflussen, im Sinne ihres Konzepts einer kontrollierten Wiederankurbelung der westdeutschen Wirtschaft zu nutzen, dürfte die französische Neigung, den deutschen Fall von der Tagesordnung der Marshallplankonferenz zu streichen, noch verstärkt haben. Mit Hinweis auf die Zuständigkeit der Außenminister versuchten französische Diplomaten jedenfalls, unerwünschte Festlegungen zu verhindern. Ihr Hauptaugenmerk richteten sie dabei nach wie vor auf die Stahl- und Metallindustrien. In einer Zwischenbilanz der Verhandlungen aus französischer Sicht von Mitte August 1947 hieß es dazu lapidar: „La sidérurgie allemande ne paraît pas nécessaire à l'équilibre du marché de l'acier." Eine vollständige Auslastung der noch brachliegenden bzw. zur Demontage vorgesehenen deutschen Kapazitäten galt angesichts der in Westeuropa ohnehin vorhandenen Überkapazitäten nicht als zwingende Notwendigkeit. Auch gegenüber der Metallindustrie verfocht der Verfasser des Memorandums einen eher restriktiven Kurs und behauptete unter Berufung auf die von den einzelnen Delegationen vorgelegten Unterlagen, daß die übrigen Länder bereit seien, die vor dem Krieg aus Deutschland bezogenen Güter selbst zu liefern. Mögliche Zeitverluste beim europäischen Wiederaufbau als Folge der Verdrängung der deutschen Konkurrenz hielt der französische Berichterstatter aus Gründen der Sicherheit für vertretbar. Schon um dieses ehrgeizige Programm nicht gänzlich unrealistisch erscheinen zu lassen, votierte er, in Abkehr von der restriktiven Linie gegenüber den Sektoren Eisen und Metall, gleichzeitig für eine Steigerung der Produktion und des Exports von Kohle und, im landwirtschaftlichen Bereich, von Kunstdünger. Die anachronistischen Züge ihrer Deutschlandpolitik schienen der französischen Diplomatie, nicht zuletzt vor dem Hintergrund der vergleichsweise guten Beziehungen zu Österreich oder Italien, gleichwohl allmählich bewußt zu werden, und die Bilanz nach vier Wochen intensiver Beratungen im Rahmen der CEEC signalisierte immerhin die Bereitschaft, diesen Anachronismus zu überwinden, freilich unter einer bestimmten Voraussetzung: „Si les travaux de la Commission avaient pour effect de hâter la disparition de 
cette anomalie, il serait essentiel qu'ils aient jeté en même temps les bases d'une structure européenne assez solide et assez cohérente pour supporter sans danger l'intégration de l'Allemagne." 69

Über das Ziel einer festen europäischen Einbindung der Westdeutschen waren sich Amerikaner und Franzosen gewiß einig; strittig war „nur“, wie dieses Ziel am ehesten erreicht werden konnte. Für die Verantwortlichen in Washington stand fest, daß eine Fortsetzung der restriktiven Politik nach dem Muster des ersten Industrieplans, wie sie der französischen Regierung noch immer vorschwebte, nicht das geeignete Mittel sein würde. Hinter den mit den Briten bereits vereinbarten Stand wollten sie auf keinen Fall zurückgehen: Den revidierten Industrieniveauplan betrachteten sie als Verhandlungsbasis; eventuelle Korrekturen im Interesse des Wiederaufbauprogramms für Europa wollten sie nur dann akzeptieren, wenn die anderen Länder ebenfalls zu Abstrichen an ihren Planungen bereit wären. ${ }^{70}$ Gerade an dieser Bereitschaft haperte es aber, denn offensichtlich herrschte unter den Teilnehmern der Marshallplankonferenz ein stillschweigender Konsens, auf eine kritische Erörterung der einzelnen Pläne und Bedarfsmeldungen zu verzichten, um keinen Einspruch gegen das jeweils eigene Programm zu provozieren.71 Im Fall der Bizone kam noch hinzu, daß die CEEC schon wegen der Zuständigkeit der Besatzungsmächte einer ernsthaften Prüfung der eingereichten Berechnungen auswich; die Delegierten Großbritanniens galten zudem auch nicht als autorisierte Vertreter bizonaler Interessen.

In dieser Situation, als es nicht mehr nur um eine möglichst effektive Nutzung der deutschen Ressourcen ging, sondern mit dem Problem einer hinreichenden Abstimmung der nationalen Einzelpläne der Erfolg des europäischen Wiederaufbauprogramms insgesamt auf dem Spiel stand, versuchten Marshall und seine Berater die Bizone als Vehikel der amerikanischen Europapolitik zu benutzen. Durch die Präsentation der bizonalen Planungen vor der CEEC, verbunden mit dem Angebot, Gegenvorschläge oder Änderungswünsche zu berücksichtigen, wollten die Amerikaner die Regierungen der teilnahmewilligen Länder ermuntern, vielleicht sogar moralisch unter Druck setzen, genauso zu verfahren. Nur auf diesem Wege, glaubten die Verantwortlichen im State Department, könne der erhoffte „cooperative approach“ der Europäer in letzter Minute noch zustande kommen. ${ }^{72}$ Mit dem Argument, die Situation der Westzonen sei ausreichend beraten worden und eine neuerliche Diskussion könne als Kritik an der Arbeit der CEEC aufgefaßt werden, blockte der britische Außenminister Bevin diesen Vorstoß jedoch ab, sicherlich auch, um eine Überprüfng der eigenen Plandaten im Lichte europäischer Erfordernisse und Möglichkeiten zu vermeiden. ${ }^{73}$ Douglas und Caffery, die in engem Kontakt mit den Pariser Verhandlungen standen, rieten daraufhin von der Präsentation ab; die Zeit bis zum 22. September, dem vorgesehenen Termin für die Verabschiedung des „vorläufigen“ CEEC-Reports, sollte statt dessen genutzt werden, um

\footnotetext{
${ }^{69}$ Note der Direction Générale Économic et Finances „Les affaires allemandes et les premiers travaux de la Commission de Cooperation Économique“ v. 15. 8. 1947 mit Begleitbrief v. 19. 8. 1947, Archives de l' Occupation Française en Allemagne et en Autriche, Colmar (künftig AO), Eco, I A 5b.

70 Acting Secretary of State an US-Botschaft Paris v. 26. 8. 1947, FRUS 1947, III, S. 383-389, hier S. 388.

$7 \mathrm{Vgl}$. dazu dic ironischen Bemerkungen Kennans in einem Bericht v. 4. 9. 1947 über seine Eindrücke von einer Paris-Reise, ebenda, S. 397-405, bes. S. $397 \mathrm{f}$.

72 Vgl. Marshall an US-Botschaft London v. 8. 9. 1947, ebenda, S. 418-419.

${ }^{73}$ Vgl. Hogan, Marshall Plan, S. 76 f.; Bullock, Bevin, S. 457-460.
} 
hinter den Kulissen auf eine angemessene Berücksichtigung Westdeutschlands zu drängen. ${ }^{74}$

Wie erfolgreich diese Bemühungen waren, läßt sich schwer beurteilen. Der „Schlußbericht" vom 22. September spiegelte nämlich, vor allem in der als Anhang beigefügten „Erklärung der beteiligten Länder, die sich mit Deutschland im Kriegszustand befunden haben", erneut jene beiden gegensätzlichen Standpunkte wider, die auch die Debatten in der CEEC und ihren Ausschüssen bestimmt hatten. ${ }^{75}$ Die enge Verknüpfung der deutschen Wirtschaft mit den übrigen europäischen Volkswirtschaften zog zwar keine der Delegationen in Zweifel, aber schon der Grundsatz, daß sich Westdeutschland nicht auf Kosten seiner künftigen Partner entwickeln dürfe, paßte mit der Feststellung, Europa werde so lange nicht „gedeihen“ können, wie die Wirtschaft der Westzonen gelähmt sei, nicht recht zusammen. Ebenso verhielt es sich mit dem französischen Anliegen, Förderung und Export von Ruhrkohle zu steigern, und der beispielsweise von den Beneluxländern favorisierten Rückkehr zu einem breitgefächerten Handelsaustausch mit dem östlichen Nachbarn. Im wesentlichen orientierte sich der Schlußbericht in seinen Westdeutschland betreffenden Teilen an den Daten des revidierten Industrieplans, sieht man einmal von der um 700000 Tonnen niedrigeren Ziffer für die Rohstahljahresproduktion der Bizone und dem ausdrücklichen Hinweis auf die Pflicht zur Steigerung des Nutzholzexports ab. Besondere Erwartungen an Westdeutschland formulierten die CEECAusschüsse sonst nur noch im Kohlensektor. Dies hing einmal damit zusammen, daß sich die Konferenz auf die Bereiche Ernährung und Landwirtschaft, Brennstoffe und Energie, Stahl und Verkehr konzentriert und Sparten wie Maschinenbau, Chemie oder Elektrotechnik weitgehend ausgeklammert hatte; zum anderen hatten sich die einzelnen technischen Ausschüsse zumeist für die Nennung von globalen Produktions- und Bedarfsziffern entschieden und auf eine Aufschlüsselung nach Ländern verzichtet.

Verglichen mit der Situation bei Beginn der Pariser Verhandlungen, als von einer Gleichbehandlung Westdeutschlands auch nicht im entferntesten die Rede sein konnte, hatte sich das Blatt am Ende zugunsten der deutschen Seite gewendet. Dank amerikanischen Drucks und wirtschaftlich motivierter Kooperationsbereitschaft solcher Länder wie Belgien und Holland entsprach die den Westzonen zugedachte Rolle bei der Rekonstruktion Westeuropas zumindest der Tendenz nach den deutschen Wünschen und Vorstellungen. Nimmt man die Zielsetzung zum Maßstab, wie sie ein britischer Diplomat wenige Tage nach Beginn der CEEC-Beratungen für die britische Delegation formuliert hatte $-~ „ 80 \%$ of our job here is how to get Germany going again quickly, but we have still to sell this to the French and others" $" 76$-, dann waren doch bemerkenswerte Fortschritte erzielt worden. Wenngleich der grundlegende Dissens zwischen den USA und Frankreich über den deutschen Part beim Marshallplan damit noch nicht überwunden war, zeigte man sich in Washington und bei der Militärregierung mit den entsprechenden Passagen des CEEC-Reports im großen und ganzen zufrieden. Eine detaillierte Un-

\footnotetext{
${ }^{74}$ Douglas an Marshall v. 12. 9. 1947, FRUS 1947, III, S. 429-430.

75 Eine deutsche Übersetzung erschien unter dem Titel: Die Wiedergesundung Europas. Schlußbericht der Pariser Wirtschaftskonferenz der Sechzehn Nationen, Teil I: Allgemeiner Teil, Oberursel (Taunus) 1948. Vgl. auch Kretzschmar, Auslandshilfe, S. 188-193; van der Beugel, Marshall Aid, S. 73, Milward, Reconstruction, S. 88; Gerd Hardach, Der Marshall-Plan. Auslandshilfe und Wiederaufbau in Westdeutschland 1948-1952, München 1994, S. 55 f.

76 Note Sanders für UK Del CEEC v. 16. 7. 1947, PRO, FO 371/62568.
} 
tersuchung der Angaben und Prognosen zu den Bereichen Elektrizität, Eisen und Stahl, Überseeverkehr, Ernährung und Landwirtschaft sowie Nutzholz, die ein Beamter der Militärregierung im Oktober 1947 vornahm, war denn auch frei von kritischen Kommentaren oder Beanstandungen. ${ }^{77}$ General Clay machte sich sogar schon Gedanken über die geeignete Form bizonaler Interessenwahrnehmung innerhalb der geplanten europäischen Organisation: Er dachte an einen kleinen britisch-amerikanischen Stab mit einem Briten oder Amerikaner als Leiter, der nach der Errichtung einer Regierung von einem deutschen Repräsentanten, unterstützt von einem allierten Berater, abgelöst werden sollte. ${ }^{78}$ So weit in die Zukunft dachte man auf deutscher Seite noch nicht; politische und wirtschaftliche Kreise versuchten erst einmal Klarheit zu gewinnen, wie sich die übrigen westeuropäischen Länder die künftige Rolle ihres Landes vorstellten und ob sich diese Vorstellungen mit den eigenen Plänen vertrugen.

\section{c) Deutsche und amerikanische Reaktionen auf den CEEC-Bericht}

Das unmittelbare Echo in Westdeutschland auf die Pariser Konferenz war schwach. Weder das bizonale „Parlament“, der Wirtschaftsrat, noch der Länderrat der US-Zone, der Zonenbeirat des britischen Besatzungsgebiets oder der Exekutivrat, die bizonale „Regierung“, diskutierten den Schlußbericht. ${ }^{79}$ Möglicherweise hing dieses Desinteresse damit zusammen, daß der neue Industrieniveauplan, der ja die Grundlage für die „deutschen" Teile des CEEC-Reports abgegeben hatte, mehrmals erörtert worden war. ${ }^{80} \mathrm{Die}$ in diesem Zusammenhang formulierte Kritik richtete sich folglich auch gegen den Pariser Schlußbericht. Hinzu kam, daß nach der Bekanntgabe der Demontageliste am 16. Oktober 1947 die öffentliche Debatte ganz von dieser neuen „Gefahr“ beherrscht wurde. ${ }^{81}$ Bei der Suche nach Gegenkonzepten spielte der Marshallplan zwar eine große Rolle: Die Pflicht zur "Wiedergutmachung“ und die Bereitschaft, „am europäischen Wiederaufbau im größten Maße mitzuwirken“, zu der sich der SPD-Politiker Fritz Henßler in einer spontanen Reaktion auf die Demontageliste bekannte ${ }^{82}$, bedingten aus deutscher Sicht geradezu den entschlossenen Widerstand gegen die Reduzierung des industriellen Potentials. Aber es war die „politische Idee“ des Marshallplans, welche die Deutschen beschworen, nicht dessen konkrete Umsetzung in ein koordiniertes europäisches Aufbauprogramm. Die Art und Weise, wie Ludwig Erhard, damals noch Leiter der „Sonderstelle Geld und Kredit“, argumentierte, war durchaus typisch: „Die Alliierten mögen Verständnis dafür haben“, schrieb er Mitte November in einem Zeitungsbeitrag, „daß das leidgeprüfte deutsche Volk bei jeder neuen Bedrängnis trotz einer der Demontageliste beigegebenen Erklärung und trotz der im Marshall-Plan verheißenen Hilfe in dem Glauben an seine Zukunft irre“ werde. „So gesehen müßten gerade die Vereinigten Staaten mit uns das größte Interesse daran haben, weitere unorganisierte Eingriffe in

\footnotetext{
77 Memoranda C. H. Price v. 9., 15., 18. u. 20. 10. 1947, alle in IfZA, MF 260, POLAD/784-4.

${ }^{78}$ Vgl. Clay an Draper v. 30. 10. 1947, Clay Papers, S. $460 \mathrm{f}$.

$79 \mathrm{Vgl}$. die in Frage kommenden Sitzungsprotokolle dieser Gremien in: AVBRD, 3.

$80 \mathrm{Vgl}$. beispielsweise 14. Sitzung Zonenbeirat brit. Zone v. 9.-11. 9. 1947, AVBRD 3, Dok. 42, S. 431-439.

81 Vgl. beispielsweise Konferenz der Ministerpräsidenten, Arbeits- und Wirtschaftsminister des Vereinigten Wirtschaftsgebietes mit dem Exekutivrat und Vertretern des Wirtschaftsrates v. 22. 10. 1947, ebenda, Dok. 75, S. 690-711; „Der Griff nach der Substanz“, Wirtschaftszeitung v. 24. 10. 1947.

82 Vgl. 15. Sitzung Zonenbeirat brit. Zone v. 15. u. 16. 10. 1947, AVBRD 3, Dok. 70, S. 674 f.
} 
das westeuropäische Wirtschaftspotential zu vermeiden. "83 Dadurch, daß die westdeutsche Wirtschaft kurzerhand zum integralen Bestandteil der westeuropäischen Wirtschaft erklärt wurde - was sie praktisch noch gar nicht war -, verwandelten sich alle Eingriffe der Besatzungsmächte in das wirtschaftliche Gefüge Westdeutschlands automatisch in Maßnahmen zum Schaden des größeren, westeuropäischen Verbunds. Die in dieser Gleichsetzung zum Ausdruck kommende, nach wie vor - allerdings nicht nur in den Westzonen - dominierende "nationale“ Sichtweise mag ein weiterer Grund für das geringe Interesse am CEEC-Bericht gewesen sein. Für manche schließlich dürfte schon das Fehlen deutscher Fachleute Anlaß gewesen sein, die Ergebnisse der Konferenz zu ignorieren. Die Vermutung der "Wirtschaftszeitung" in einem Bericht über den Abschluß der Pariser Marshallplankonferenz, man könne in „Deutschland ... die ganze Bedeutung dieses nunmehr vollzogenen Tatbestandes und seiner notwendigen Folgen im Verhältnis zu den Ländern Westeuropas und zu Amerika" noch gar nicht erfassen ${ }^{84}$, traf mithin zweifellos zu, wenngleich die Gründe für dieses mangelhafte Verständnis unterschiedlicher Art waren.

Einer der wenigen Experten, welche die Bedeutung der Pariser Verhandlungen und des CEEC-Reports erkannt hatten, war der bereits erwähnte Hauptgeschäftsführer der Wirtschaftsvereinigung Eisen- und Stahlindustrie, Wilhelm Salewski. In einem kleinen Aufsatz in der Fachzeitschrift „Stahl und Eisen“ setzte er sich ausführlich mit den Konsequenzen des Schlußberichts für "seine" Branche auseinander, verbunden mit der ausdrücklichen Warnung, daß die „Tatsache, daß die deutsche Eisenindustrie heute ... gewissermaßen nur als Objekt am Rande des Geschehens" stehe, auf keinen Fall dazu verführen dürfe, „die gegenwärtigen Vorgänge zu unterschätzen oder ihnen infolge der gewiß nicht geringen unmittelbaren Sorgen etwa die Aufmerksamkeit zu ersparen, die sie gewißlich" verdienten. ${ }^{85}$ Getreu dieser Devise war für Salewski schon die gegenüber dem revidierten Industrieniveauplan deutlich geringere Produktionsvorgabe von 10 Mill. Tonnen für das Jahr 1951 Anlaß zur Kritik, und zwar nicht zuletzt deshalb, weil diese Menge „nicht einmal ein Fünftel“ der Planzahl für die Teilnehmer ausmachte, während die deutsche Stahlindustrie vor dem Kriege „etwa $40 \%$ der Erzeugung ganz Europas" hervorgebracht hatte. Die vorgesehene Obergrenze schloß, da bedurfte es gar keiner ausdrücklichen Erwähnung, eine angemessene Berücksichtigung der westdeutschen Stahlindustrie seiner Ansicht nach von vornherein aus. Anstatt die dort vorhandenen Produktionsanlagen zu nutzen, müßten nämlich in den übrigen Ländern die Kapazitäten beträchtlich erweitert werden, um wie geplant 1951 den Vorkriegsstand erreichen zu können. Die amerikanische Kritik an den „zu weit gesteckten Ausbauplänen“ fand deshalb verständlicherweise den Beifall des Verbandsfunktionärs. Skeptisch beurteilte Salewski aber auch die CEEC-Planungen für die Rohstoffversorgung und den Einfuhrbedarf der Teilnehmerländer insgesamt, wobei er zumindest im zweiten Fall erkennen ließ, daß eine bessere Ausnutzung der westdeutschen Möglichkeiten Engpässe gar nicht erst entstehen lassen oder wenigstens abmildern würde. Trotz dieser alles in allem eher nega-

${ }^{83}$ Ludwig Erhard, Deutung der Demontage, „Wirtschaftsspiegel“ v. 15. 11. 1947, abgedruckt in: Karl Hohmann (Hrsg.), Ludwig Erhard. Gedanken aus fünf Jahrzehnten. Reden und Schriften, Düsseldorf u. a. 1988 , S. $83-90$, hier S. 84 u. 88.

${ }^{84}$ "Hilfe und Selbsthilfe", Wirtschaftszeitung v. 3. 10. 1947.

85 "Wilhelm Salcwski, Die europäische Eisenwirtschaft im Zeichen des Marshall-Planes, in: Stahl und Eisen 68 (1948), S. 15-19; dort auch die folgenden Zitate. 
tiven Bilanz würdigte Salewski die Pariser Verhandlungen jedoch als „die erste internationale Zusammenkunft der Eisenindustrie seit dem Kriege“, die „für alle Beteiligten von großem Wert" gewesen sei.

$\mathrm{Zu}$ einem ähnlichen Urteil gelangte auch ein Bericht im offiziellen Organ der bizonalen Verwaltung für Wirtschaft: Die Freude darüber, daß die „Bedeutung der Bizone für den Wiederaufbau Europas voll“ anerkannt werde, wurde allerdings getrübt durch das Eingeständnis, im Bericht selber sei davon wenig zu spüren. Der Haupteinwand richtete sich ebenfalls gegen die Höhe der Stahlerzeugung, die „keinesfalls an das als notwendig Erkannte auch nur annähernd" heranreiche und deshalb verhindere, daß die eisenverarbeitende Industrie Westdeutschlands einen „so großen Anteil am Aufbau Europas“ nehme, wie es „ihrer Kapazität nach durchaus möglich wäre“. ${ }^{86}$

Die wohl massivste öffentliche Kritik formulierte jedoch Fritz Baade, SPD-Mitglied und seit April 1948 Direktor des renommierten Instituts für Weltwirtschaft in Kiel, der nach dem Krieg mehrere Monate in den USA verbracht hatte. In der Einleitung zu einer Dokumentation ausgewählter Abschnitte aus den Berichten des Harriman- und des Herter-Komitees, die zusammen mit weiteren von Präsident Truman eingesetzten Ausschüssen den europäischen Vorschlag analysiert und Empfehlungen zur Umsetzung des Marshallplans erarbeitet hatten, attackierte Baade mit ungewohnter Schärfe das CEECProgramm. ${ }^{87}$ Mit dem Entschluß, die Wahrnehmung westdeutscher Interessen Mitgliedern der Militärregierungen anzuvertrauen, war für ihn eine Benachteiligung der Westzonen bereits programmiert. Es könne „nicht wundernehmen, daß das Fehlen autorisierter deutscher Vertreter und Sachverständiger zu einer einseitigen und schiefen Planung des westdeutschen Wiederaufbaus geführt" habe. Die wirtschaftspolitische Absicht der sechzehn europäischen Nationen und der Militärregierungen erblickte er darin, mit Hilfe des Industrieniveauplans und des darauf basierenden CEEC-Programms die westdeutsche Ausfuhr von Stahl, Walzwerkerzeugnissen, schweren Maschinen und Produkten der Schwerchemie weitgehend zu unterbinden und das frühere industrielle Zentrum Europas in ein Rohstoffexportland zu verwandeln. Folge dieser verfehlten Planung waren die seiner Ansicht nach "geradezu phantastischen Mengen“ an Rohstahl, Halbzeug und Stahlerzeugnissen auf der „Einkaufsliste“ des Pariser Komitees: Nicht weniger als 5,8 von insgesamt veranschlagten $\mathrm{rd}$. $21 \mathrm{Mrd}$. Dollar an Hilfsleistungen waren dafür vorgesehen. Auf diesem Wege, so Baade, hofften die sechzehn CEEC-Mitgliedsländer, „in der Weltwirtschaft so schnell wie möglich jeden durch die Ausschaltung der deutschen Lieferungen frei werdenden Platz zu besetzen“. Die Kehrseite der Stahlbedarfsmeldungen war für ihn die einseitige Festlegung Westdeutschlands auf Kohlenexporte, die den Pariser Planern die Möglichkeit eröffne, „von Jahr zu Jahr in rascher Progression auf amerikanische Kohlenlieferungen zu verzichten“. Immerhin, so konstatierte Baade mit einer gewissen Befriedigung, habe der „wesentliche amerikanische Beitrag zur Marshall-Plan-Diskussion“ zwischen Herbst 1947 und Frühjahr 1948 darin bestanden, diese „Fehlplanungen des Pariser Gutachtens zu korrigieren“.

86 „Der Anteil der Bizone am ERP. Über die Unterlagen der Pariser Verhandlungen“, ungezeichneter Artikel in: Wirtschaftsverwaltung 1 (1948), Heft 8, S. 19-21.

${ }^{87}$ Deutschlands Beitrag zum Marshall-Plan. Ausgewählte Kapitel aus den Harriman- und Herter-Reports. Mit einer Einleitung v. Fritz Baade, Hamburg 1948; dort auch die folgenden Zitate. Im Sinne Baades auch Kretzschmar, Auslandshilfe, bes. S. $190 \mathrm{f}$. Zu den verschiedenen Komitees vgl. van der Beugel, Marshall Aid, S. 87-94; Brähler, Marshallplan, S. 190-192; Hardach, Marshall-Plan, S. 57-61. 
Selbst wenn man in Rechnung stellt, daß Baade dank seiner intensiven Kontakte in die USA recht gut über die dortige Stimmung informiert war und deshalb sicher sein konnte, in der Sache bei vielen Politikern und Geschäftsleuten „offene Türen einzurennen“, überrascht der aggressive Ton und die Undifferenziertheit seiner Kritik. Nicht einmal die Sprecher unmittelbar betroffener Industriezweige ließen sich in der Öffentlichkeit zu solchen „ungerechten" Pauschalurteilen hinreißen wie der sozialdemokratische Wirtschaftsexperte aus Kiel. Schließlich hatten sich insbesondere die amerikanische Militärregierung und während der Pariser Verhandlungen die Delegationen Großbritanniens und der Beneluxstaaten für eine kontrollierte Rekonstruktion der früheren wirtschaftlichen Strukturen und damit der traditionellen Rolle der deutschen Industrie eingesetzt. Diese Verdienste ignorierte Baade aufgrund seiner maximalistischen Denkweise jedoch völlig. Auf die „europäische Dimension“ des CEEC-Berichts ging er deshalb erst gar nicht ein. In diesem Punkt unterschied er sich allerdings kaum von anderen deutschen Reaktionen. Die Frage, ob die skizzierten Schritte in Richtung Wiederaufbau in Europa überhaupt erfolgversprechend, ob insbesondere die Vorschläge zur Vertiefung der wirtschaftlichen Zusammenarbeit diesem Ziel überhaupt dienlich waren, interessierte allenfalls am Rande. Die Auseinandersetzung mit dem Problem der europäischen Kooperation oder gar Integration hatte auf deutscher Seite noch gar nicht begonnen.

Dies war in den Vereinigten Staaten anders. Vor allem zwei der eigens von der Regierung eingesetzten Ausschüsse, das Harriman-Komitee und das Herter-Komitee, beschäftigten sich eingehend mit den Auswirkungen des Hilfsprogramms auf die westeuropäischen Volkswirtschaften in ihrer Gesamtheit und seinen Rückwirkungen auf die heimische Wirtschaft. ${ }^{88}$ Gleichwohl widmeten beide Ausschüsse der Rolle Westdeutschlands größte Aufmerksamkeit. Das vom damaligen Handelsminister und späteren Sonderbeauftragten der Economic Cooperation Administration (ECA) für Europa, W. Averell Harriman, geleitete Komitee, dem auch der künftige ECA-Administrator Paul G. Hoffman angehörte, wertete die Hilfe für den Wiederaufbau der Industrie, der Landwirtschaft und des Transportwesens in den Westzonen als den entscheidenden Beitrag zum Wiederaufbau Westeuropas. Das Komitee plädierte in seinem Abschlußbericht deshalb für eine Umverteilung der Zuwendungen mit dem Ziel, den Westdeutschland zugedachten Hilfsbetrag über die in Paris vorgeschlagene Summe hinaus zu erhöhen. Der nach dem Zweiten Vorsitzenden Christian A. Herter benannte Hauptausschuß des Repräsentantenhauses für auswärtige Hilfe kam in seinem Deutschlandbericht vom 6. Februar 1948 ebenfalls zu dem Ergebnis, daß die im CEEC-Bericht für Westdeutschland vorgesehenen Hilfsmaßnahmen wegen der großen Bedeutung seiner Industrie nicht ausreichend seien. Beide Berichte erwähnten allerdings ausdrücklich, und darin unterschieden sie sich eben doch von den deutschen Stellungnahmen, die historischen Ursachen der deutschen Misere: „Zweimal innerhalb eines Menschenalters“, hieß es beispielsweise in der entsprechenden Länderstudie des Herter-Komitees, habe „Deutschland unter dem Einfluß einer unheilvollen Führung Europas Stabilität und den Weltfrieden gestört“ ${ }^{89}$ Beide Expertenteams akzeptierten das Sicherheitsbedürfnis der europäischen Nachbarn und die auch in den Vereinigten Staaten verbreitete Überzeugung, daß der

\footnotetext{
${ }^{88}$ Vgl. Brown/Opie, Foreign Assistance, S. 136-139; John C. Campbell, The United States in World Affairs 1947-1948, New York - London 1948, bes. S. 480-483; Hardach, Marshall-Plan, S. 57-65.

${ }^{89}$ Zit. n. der dt. Übers. in: Deutschlands Beitrag zum Marshall-Plan, S. 30.
} 
wirtschaftliche Wiederaufbau in dem besetzten Land nur unter schärfster alliierter Kontrolle vonstatten gehen durfte: Den revidierten Industrieniveauplan, der insbesondere Baade ein Dorn im Auge war, verteidigte das Harriman-Komitee folglich, bei aller Kritik am CEEC-Schlußbericht, als angemessene Planungsgrundlage. Die Erhöhung des deutschen Anteils an den geplanten Hilfsmaßnahmen auf Kosten der übrigen Teilnehmer - eine Forderung, die während der „Hearings" und Beratungen im Kongreß und im Senat verschiedentlich Unterstützung fand - unterblieb schließlich. Die im Zuge der Reduzierung des ERP-Gesamtvolumens erforderlichen Einsparungen trafen die Westzonen nicht weniger als die anderen Empfängerländer; die im ersten OEEC-Jahresplan 1948/49 angeforderten bzw. genehmigten Mittel waren gegenüber den Voranschlägen sogar überdurchschnittlich stark gekürzt.

Tabelle 3: Defizite und geplante ERP-Anteile ausgewählter Länder (in Mill. Dollar)

Land

Defizite gegenüber dem amerik. Kontinent im Jahr 1948 lt. CEEC-Berichta)

Bizone

Fr. Zone

Frankreich

Großbritannien

Italien

Niederlande
1150

120

1760

2630

930

630
ERP-Beträge für die ersten 15 Monate lt.

Vorschlag des State

Departments vom 20. 1. 1948b)

Quellen: a) Wiedergesundung Europas, S. 23.

b) Das Europäische Wiederaufbauprogramm der Vereinigten Staaten, in: Europa-Archiv 3 (1948), S. 1375.

c) Wexler, Marshall-Plan, S. 62.

Während für viele - zeitgenössische und spätere - deutsche Kommentatoren das „europäische Element" der CEEC-Planungen und des Marshallplans überhaupt nahezu identisch war mit der optimalen Berücksichtigung deutscher Interessen ${ }^{90}$, überwog in den Nachbarländern und in den Vereinigten Staaten also eine andere, entgegengesetzte Sicht: Die Schaffung europäischer Instanzen verstand man dort als Schutz vor neuen Hegemoniebestrebungen der „unruhigen Deutschen“. Die Vereinigten Staaten, so versicherte Präsident Truman in seiner Kongreßbotschaft zum europäischen Wiederaufbauprogramm vom 19. Dezember 1947, seien entschlossen, „Deutschland niemals wieder Europa beherrschen oder den Frieden der Welt bedrohen zu lassen". ${ }^{91}$ Selbst wenn es in der amerikanischen Administration und Öffentlichkeit Leute geben mochte, die solche Befürchtungen mittlerweile als übertrieben und überholt ansahen - in Europa nahm man diese Gefahren weiterhin ernst. Die Aufnahme einschlägiger Daten in den CEECReport und der prinzipielle Konsens über die hohe Bedeutung des westdeutschen Wirtschaftspotentials implizierten deshalb nicht für alle westeuropäischen Regierungen ei-

\footnotetext{
90 Typische Beispiele sind: Deutschlands Beitrag zum Marshall-Plan und Kretzschmar, Auslandshilfe, bes. S. $189 \mathrm{f}$.

${ }^{9}$ Zit. n. der dt. Übers. in: Europa-Archiv 3 (1948), S. 1266-1272, hier S. 1270.
} 
nen gleichberechtigen Status der drei Westzonen innerhalb der geplanten internationalen Organisation.

\section{Die Entscheidung über eine deutsche Beteiligung am European Recovery Program}

Die aktive Mitwirkung der westlichen Besatzungszonen an der Erstellung eines Hilfsprogramms hatte sich bis zu diesem Zeitpunkt darauf beschränkt, die CEEC-Fragebogen zu beantworten. Eine direkte Repräsentation in Paris hatte es nicht gegeben; die Bizone war lediglich indirekt, durch Mitglieder der britischen Control Commission for Germany, welche der Delegation Großbritanniens angehörten, vertreten gewesen. Vor allem die amerikanische Seite drängte infolgedessen auf eine uneingeschränkte Beteiligung der Westzonen am ERP.

Falls die französische Regierung noch gehofft haben sollte, die Aufnahmebedingungen für Westdeutschland unter das Niveau des revidierten Industrieplans drücken zu können, so mußte sie diese Hoffnung spätestens während der Londoner Außenministerkonferenz vom November und Dezember 1947 aufgeben. Der Rat einigte sich nämlich darauf, für alle vier Zonen eine Höchstproduktion von 11,5 Millionen Tonnen Rohstahl pro Jahr zuzulassen. Auf Bidaults Wunsch bekräftigten Bevin und Marshall, daß der deutsche Wiederaufbau gegenüber der Rekonstruktion der anderen Teilnehmerländer keine Priorität genießen dürfe. ${ }^{92}$ Die frühere Forderung nach Vorrang der „demokratischen Länder" war damit auf die Maxime zusammengeschrumpft, den einstigen Aggressor wenigstens nicht zu bevorzugen.

An der Notwendigkeit eines beschleunigten wirtschaftlichen und politischen Wiederaufbaus bestand jedoch zumindest auf seiten der Amerikaner kein Zweifel mehr, nachdem der Abbruch der Londoner Außenministertagung das vorläufige „Aus“ für gemeinsame deutschlandpolitische Schritte der vier Besatzungsmächte signalisiert hatte. Um so dringlicher erschien nun die Schaffung westeuropäischer Strukturen und die feste, vertragliche Einbindung der Westzonen in die im Entstehen begriffene Organisation. Es kam darauf an, das Verhältnis zwischen dem westlichen Verlangen nach größtmöglicher Sicherheit vor „Deutschland“ einerseits und nach einem größtmöglichen Beitrag Westdeutschlands zur Wiedergenesung Europas andererseits neu zu definieren. Diesem Zweck dienten die Londoner Verhandlungen der drei Westmächte vom Februar und März 1948, zu denen zeitweise auch die Beneluxländer hinzugezogen wurden. ${ }^{93}$

Eines der wichtigsten Themen neben Ruhrkontrolle, Reparationen und dem künftigen politischen Aufbau war die Einbeziehung der Westzonen in das Wiederaufbauprogramm und die Mitarbeit in der geplanten permanenten Organisation. Bereits in den zweiseitigen Vorgesprächen im Foreign Office machten die amerikanischen Unterhändler ihre Kollegen mit den Absichten ihrer Regierung bekannt: „Full partnership of Western zones in ERP, including participation in any continuing CEEC organization, particular efforts at economic cooperation within ERP area such as study of possibilities

92 US-Delegation an Truman v. 11. 12. 1947, FRUS 1947, II, S. 765 f.; das Zitat in Anm. 52.

93 Vgl. Milward, Reconstruction, S. 144 f.; Hogan, Marshall Plan, S. 129-132; Krieger, Clay, S. 311-336;

Die Londoner Verhandlungen sind dokumentiert in FRUS 1948, II, S. 75-145. 
of customs union, in multilateral clearing arrangements etc." Dieser Zielsetzung stimmten die britischen Diplomaten ebenso zu wie der naheliegenden praktischen Konsequenz, den deutschen Behörden größere Mitspracherechte bei der Programmplanung und -umsetzung zuzugestehen. ${ }^{94}$ Grundsätzliche Einwände von französischer Seite waren nach diesem amerikanisch-britischen Schulterschluß kaum mehr zu erwarten. Bereits am zweiten Tag der Konferenz, am 24. Februar 1948, sprach der US-Botschafter in London, Lewis W. Douglas, der zugleich als Delegationschef fungierte, die Rolle Westdeutschlands im Rahmen des Marshallplans an. Um seine Verhandlungspartner für die amerikanische Konzeption zu gewinnen, unterstrich er die veränderte Stellung „Deutschlands“, verglichen mit früheren Zeiten: „stripped of territories, smaller population, must export more if to be economically self contained, actually impotent and will remain so except as instrumentality of some other power." Auf seine Mitwirkung könne jedoch um der politischen und wirtschaftlichen Stabilität Westeuropas willen nicht verzichtet werden. Am liebsten wäre ihm eine einheitliche Vertretung der drei Westzonen gewesen; bis es soweit war, sollten die Bizone und die französische Zone mit jeweils einer Stimme an der geplanten Organisation beteiligt und die Aktivitäten nach Möglichkeit koordiniert werden. Nachdem die britische Seite erwartungsgemäß ihr grundsätzliches Einverständnis signalisiert hatte, sicherte auch der französische Botschafter und Chefunterhändler René Massigli eine positive Prüfung des amerikanischen Vorschlags zu: „His government wanted Germany to become (a) useful member (of the) European community in which it must be integrated but problem required caution as experience under Hitler showed necessity bringing Germany into Europe rather than building Europe around Germany. "Zum Studium des amerikanischen Vorschlags wurde eigens eine Arbeitsgruppe eingesetzt. ${ }^{95}$

Zwar ließ sich die französische Delegation auch durch unmißverständliche Drohungen mit einer eventuellen Kürzung der zu erwartenden ERP-Mittel nicht zu einer verbindlichen Erklärung in Sachen Zonenfusion bewegen ${ }^{96}$, doch die Frage der Einbeziehung der Westzonen konnte zur Zufriedenheit der Amerikaner gelöst werden. Am 2. März einigten sich die Delegationen der USA, Großbritanniens und Frankreichs auf einen von der Arbeitsgruppe vorgeschlagenen Sechs-Punkte-Katalog, der unter anderem vorsah, daß die Bizone und die französische Zone als unabhängige Teilnehmer gelten und demzufolge das geplante multilaterale sowie das zweiseitige Abkommen mit den Vereinigten Staaten jeweils selbst unterzeichnen sollten. Die Arbeitsgruppe votierte für die Vollmitgliedschaft, die allerdings auf Regierungsebene zunächst von alliiertem Personal wahrgenommen werden sollte. Immerhin wurde den Besatzungsbehörden nahegelegt, deutsche Berater und Experten an den künftigen Arbeiten in Paris zu beteiligen.97 Im Konferenz-Schlußkommuniqué vom 6. März 1948 bekräftigten die drei Westmächte diesen Beschluß. Um den Eindruck zu vermeiden, als handelten sie über die Köpfe der kleineren europäischen Länder hinweg, sollte die Entscheidung über die Modalitäten

\footnotetext{
94 Douglas an Sec State v. 21. 2. 1948, FRUS 1948, II, S. 78-79, hier S. 79.

95 Douglas an Sec State v. 25. 2. 1948, FRUS 1948, II, S. 87-89. Vgl. auch Vermerk Nicholls: „Western Germany and E.R.P." v. 25. 2. 1948, PRO, FO 371/70732.

${ }^{96}$ Zum Einsatz der ERP-Hilfe als politisches Druckmittel vgl. Douglas an Sec State v. 1. 3. 1948, FRUS 1948, II, S. 107.

${ }^{97}$ Douglas an Sec State v. 2. 3. 1948, ebenda, S. 116-117.
} 
der Mitarbeit der Besatzungszonen dem nächsten CEEC-Treffen vorbehalten bleiben. ${ }^{98}$ Für einen ersten „Auftritt“ schon am 15. März, den Bevin, Douglas und Massigli zeitweilig in Betracht gezogen hatten, war es deshalb zu spät.99

Ebenso wie das State Department war auch die britische Diplomatie sorgsam darauf bedacht, die übrigen CEEC-Länder nicht durch ein zu rasches oder eigenmächtiges Vorgehen zu brüskieren. Um die Aufnahme der Bizone und der französischen Zone als 17. und 18. Teilnehmer - mit den gleichen Rechten und Pflichten wie die Gründungsmitglieder - nicht zu gefährden, plädierte das Foreign Office sowohl in der Frage der einheitlichen Vertretung der Westzonen als auch im Fall von Protesten gegen eine Beteiligung deutscher Experten für einen behutsamen Kurs. Obwohl sie von der Notwendigkeit einer solchen Mitarbeit schon aus rein praktischen Gründen überzeugt waren, wollten die Briten nichts überstürzen: „Our intention would rather be to infiltrate German advisers gradually to the extent that this can be done without giving rise to objections from other countries." 100

Auf der zweiten CEEC-Vollversammlung am 15. und 16. März 1948 präsentierte der britische Außenminister Bevin, wie in London vereinbart, den Antrag auf Einbeziehung der Westzonen. ${ }^{101}$ Bereits am ersten Konferenztag nahm mit Bidault der einflußreichste potentielle Kritiker dieses Vorhabens für die „Aufnahme“ Westdeutschlands Stellung: „Par delà le nationalisme, nous offrons à l'Allemagne l'occasion d'éprouver ce sentiment de solidarité fraternelle qu'elle a souvent méprisé. “102 Bidaults Erklärung hatte Signalcharakter. Die Konferenz nahm den Antrag am 16. März einstimmig an, wobei die Zustimmung manchen Delegationen sicherlich dadurch erleichtert worden sein dürfte, daß auf der „politischen“ Ebene zunächst alliierte Repräsentanten die deutschen Interessen wahrnehmen würden. Mit diesem Aufnahmeverfahren hatten die drei Westmächte auf mögliche Empfindlichkeiten der kleineren Länder Rücksicht genommen und die Autorität der CEEC respektiert. Mehr als eine diplomatische Geste war dies zumindest aus amerikanischer Sicht freilich nicht: Bereits am 17. Februar 1948 hatte das Zweimächtekontrollamt den Vorsitzenden des bizonalen Exekutivrates nämlich zur Mitarbeit an der Formulierung des Wiederaufbauplans aufgefordert. ${ }^{103}$ Auf deutscher Seite wurde dieses Schreiben als eine Art Bestätigung gewertet, daß die Bizone in den Marshallplan einbezogen werde ${ }^{104}$ - gewiß zu Recht, denn die Teilnahme der Westzonen am ERP hätte die westliche Vormacht auch gegen Widerstände auf der Londoner Sechsmächtekonferenz oder der CEEC-Tagung durchgesetzt. Offen waren lediglich Art und Umfang der Beteiligung; hier zeigten sich die amerikanischen Planer durchaus konzessionsbereit.

Ist angesichts dieser starken Abhängigkeit der Westzonen von den Besatzungsmächten der eingangs verworfene Begriff des „penetrierten Systems“ nicht doch angemessen?

\footnotetext{
${ }^{98}$ Kommuniqué v. 6. 3. 1948, ebenda, S. 141-143. Vgl. auch Marshall an Sebald v. 5. 3. 1948, FRUS 1948, III, S. 388.

${ }^{99}$ Douglas an Sec State v. 2. 3. 1948, FRUS 1948, II, S. 114-116.

120 Foreign Office-Note: „Participation of Western Germany in the C.E.E.C." o. D. (Empfangsbestätigung v. 15. 3. 1948), PRO, FO 371/70732.

101 Vgl. Die Konferenzen des CEEC im Rahmen des curopäischen Wiederaufbauprogramms, in: EuropaArchiv 3 (1948), S. 1305-1316.

102 „Résumé du Discours prononcé par M. Georges Bidault“ v. 15. 3. 1948, MAE, Y 1944-1949, Vol. 131.

${ }_{103} \mathrm{BICO}$ an Vors. Exekutivrat v. 17. 2. 1948, BA, NL Pünder, 704.

${ }^{104}$ Sitzung Exekutivrat v. 17. 2. 1948, AVBRD 4, bearb. v. Christoph Weisz, Hans-Dieter Kreikamp u. Bernd Steger, München - Wien 1983, Dok. 30, S. $343 \mathrm{f}$.
} 
Gewiß ließe sich argumentieren, daß ein Besatzungsregime den Extremfall eines solchen Dependenzverhältnisses darstelle. Bemerkenswert erscheint indes, daß insbesondere die Amerikaner, ungeachtet aller Bemühungen, Kontrollmechanismen und "Sicherungen“ zu installieren, auf eine möglichst rasche, wenngleich zunächst begrenzte Eigenverantwortlichkeit der Deutschen drängten. Mit anderen Worten, das Stadium der Besatzungsherrschaft sollte nicht länger als unbedingt nötig aufrechterhalten werden. Oberflächlich betrachtet, mag die Beziehung zwischen den Westmächten, allen voran die USA, und Westdeutschland durchaus Züge eines penetrierten Systems aufweisen - im Kern ging es jedoch um die Rekonstruktion und gleichzeitige Integration eines den meisten anderen westeuropäischen Ländern an Autonomie prinzipiell ebenbürtigen Staates.

Den Westdeutschen ermöglichte die Teilnahme am Marshallplan denn auch die ersten Schritte aus der außenpolitischen Isolation und damit aus dem „Schatten des Dritten Reiches“. Manchen ging diese Entwicklung viel zu langsam. Diejenigen mit dem notorisch guten Gewissen hatten die Vergangenheit bereits „bewältigt" und konnten das Zögern der Siegermächte und die Befürchtungen der Nachbarländer gar nicht begreifen. Aber auch einige derjenigen, die es sich nicht so einfach machten, bewiesen erstaunlich wenig Gespür im Umgang mit den ehemaligen Gegnern und Opfern Nazi-Deutschlands. Lautstarke Polemik übertönte oft die nüchterne Bestandsaufnahme. Dabei hatte doch die amerikanische Regierung, unterstützt von Großbritannien und den Beneluxländern, bereits die Voraussetzungen dafür geschaffen, daß Westdeutschland zumindest in wirtschaftlicher Hinsicht an die frühere Rolle des Deutschen Reiches anknüpfen konnte. Zwar war man noch weit entfernt von der einstigen industriellen Machtposition, doch die Zeichen standen, keine drei Jahre nach Kriegsende, günstig. 